MATHEMATICS OF COMPUTATION

Volume 81, Number 277, January 2012, Pages 191-218

S $0025-5718(2011) 02491-9$

Article electronically published on June 28, 2011

\title{
UNIFORM-IN-TIME ERROR ESTIMATES FOR SPECTRAL GALERKIN APPROXIMATIONS OF A MASS DIFFUSION MODEL
}

\author{
J. V. GUTIÉRREZ-SANTACREU AND M. A. ROJAS-MEDAR
}

\begin{abstract}
The goal of this paper is to present uniform-in-time error estimates by considering spectral Galerkin approximations of the Kazhikhov-Smagulov model for strong solutions. To be more precise, we derive an optimal uniformin-time error bound in the $\boldsymbol{H}^{1} \times H^{2}$ norm for the velocity and density approximations being stated in Theorem 6 .
\end{abstract}

\section{INTRODUCTION}

There is a wide range of phenomena that the classical Navier-Stokes equations cannot describe, above all, when modeling fluids with nonconstant density. Therefore, it is easy to understand the importance of studying models which take into account a time-dependent density. In this paper we are particularly interested in the mixture of two fluids in the presence of a mass diffusion effect called Fick's law. This model is also known as the Kazhikhov-Smagulov model.

1.1. Model. Let $\Omega \subseteq \mathbb{R}^{d}$ ( $d=2$ or 3 ) be a bounded domain with boundary $\Gamma$ of class $C^{2,1}$. Denote by $Q=\Omega \times(0, \infty)$ the space-time cylindrical domain, and $\Sigma=$ $\Gamma \times(0, \infty)$ its boundary. The equation of the motion of a viscous, incompressible fluid subject to a mass diffusion effect, say $\lambda>0$, can be written as:

$$
\left\{\begin{array}{rlrl}
\rho\left(\partial_{t} \boldsymbol{u}+\boldsymbol{u} \cdot \nabla \boldsymbol{u}\right)-\mu \Delta \boldsymbol{u}+\nabla p & & \\
-\lambda((\boldsymbol{u} \cdot \nabla) \nabla \rho+(\nabla \rho \cdot \nabla) \boldsymbol{u}) & =\rho \boldsymbol{f} & \text { in } Q, \\
\nabla \cdot \boldsymbol{u} & =0 & \text { in } Q, \\
\partial_{t} \rho-\lambda \Delta \rho+\boldsymbol{u} \cdot \nabla \rho & =0 & & \text { in } Q .
\end{array}\right.
$$

The unknowns are $\boldsymbol{u}: \Sigma \rightarrow \mathbb{R}^{d}$, the fluid velocity, $p: \Sigma \rightarrow \mathbb{R}$, the pressure, and $\rho: \Sigma \rightarrow \mathbb{R}^{+}$, the fluid density. The function $\boldsymbol{f}$ is the external volume forces applied to the fluid confined in $\Omega$, and $\mu>0$ is the kinematic viscosity.

To equations (1) we append the boundary conditions

$$
\boldsymbol{u}(\boldsymbol{x}, t)=0, \quad \partial_{\boldsymbol{n}} \rho(\boldsymbol{x}, t)=0 \quad \text { for all } \quad(\boldsymbol{x}, t) \in \Sigma,
$$

Received by the editor August 31, 2009 and, in revised form, October 9, 2010.

2010 Mathematics Subject Classification. Primary 35Q35, 65M12, 65M15, 76D05.

Key words and phrases. Fluids with diffusion, spectral Galerkin method, error estimates.

The first author's work was partially supported by project MTM2006-07932, Spain.

The second author's work was partially supported by project MTM2006-07932, Spain and Grant 1080628, Fondecyt-Chile. 
and the initial conditions

$$
\boldsymbol{u}(\boldsymbol{x}, 0)=\boldsymbol{u}_{0}, \quad \rho(\boldsymbol{x}, 0)=\rho_{0} \quad \text { for all } \quad \boldsymbol{x} \in \Omega,
$$

where $\boldsymbol{n}$ is the unit outwards normal vector on $\Gamma$. In this case, the initial density is assumed to hold:

$$
0<\alpha \leq \rho_{0}(\boldsymbol{x}) \leq \beta<\infty \quad \text { for all } \quad \boldsymbol{x} \in \Omega .
$$

An extensive physical discussion and a derivation of problem (1) can be seen in Frank and Kamenestskii [10], Kazhikhov and Smagulov [19], and Antoncev, Kazhikhov and Monakhov [2].

At this point it is well to emphasize the main mathematical difficulties related to (11). All of the difficulties one finds when studying numerically and/or theoretically the Navier-Stokes equations are presented in model (11). Furthermore, we can find the following.

(1) The coupling of the density with the convective and the velocity time derivative term, respectively.

(2) The presence of new "convective" terms.

(3) The density must satisfy a maximum principle.

The maximum principle causes no additional difficulties in the previous works 4, 11, 9, 19, 27, 30, 29. This is due to the fact that semi-Galerkin approximations are used. These approximations utilize a proper finite dimensional space to approximate the weak formulation of the momentum equation, and the mass equation remains pointwisely. This means that the previous works are nonviable to design numerical schemes. In [8] assuming regularity of the data and solution (not necessarily stable) of problem (11), which does not require compatibility conditions at time $t=0$ [16], Damázio and Rojas-Medar proved error estimates of the form

$$
\left\|\nabla\left(\boldsymbol{u}-\boldsymbol{u}^{k}\right)(t)\right\|+\left\|\Delta\left(\rho-\rho^{k}\right)(t)\right\| \leq \frac{K(t)}{\lambda_{k+1}},
$$

where $K(t)$ is a function which grows exponentially with time, and $\lambda_{k+1}$ is the $(k+1)^{t h}$ eigenvalue of the Stokes operator. Such a bound is meaningless as $t \rightarrow \infty$ since $\frac{K(t)}{\lambda_{k+1}} \rightarrow \infty$ for a fixed pair $\left(\rho^{k}, \boldsymbol{u}^{k}\right)$ being the approximation computed by means of a semi-Galerkin method.

In view of the above discussion, our goal is then to derive uniform-in-time error estimates for a conditionally asymptotically stable solution of (11) by using fully spectral Galerkin approximations based on the eigenvalues and eigenfunctions provided by the Stokes operator for the velocity and the Poisson-Neumann operator for the density. It is worth mentioning that this is the first work, to our knowledge, to use a fully spectral Galerkin method for approximating model (1).

1.2. Outline. We finish this section with an overview of the main contributions for model (1) and the notation used below. In Section 2, we introduce the concept of perturbations associated with model (1) and state the suitable hypotheses on the perturbations and the solution to be approximated. Finally, we give the main result of our work. In Section 3, we establish a priori estimates for solution and perturbation. Moreover, we give the approximate maximum principle for the density, some fundamental differential inequalities between perturbations, and approximate solutions. Finally, by contradiction, we prove the main result. 
1.3. Known results. For model (1), Kazhikhov and Smagulov 19 proved, using a semi-Galerkin method, the existence of global-in-time weak and local-in-time strong solutions under hypothesis (4) and the assumption about the viscosity and diffusion coefficient: $\lambda<2 \mu /(\beta-\alpha)$. Also, via this method, Salvi [27] proved the globalin-time existence of weak solutions in cylindrical and noncylindrical domains in $\mathbb{R}^{n}$ ( $n$ arbitrary) under the same condition on the coefficients of viscosity and diffusion as in [19. In 3] the existence of a local-in-time, unique, strong solution is proved for the Euler diffusion model, i.e., $\mu=0$ in (1). Clearly, the boundary condition $\boldsymbol{u}=0$ on $\Gamma$ is replaced by $\boldsymbol{u} \cdot \boldsymbol{n}=0$ on $\Gamma$. After this result one realizes that, in the viscous case, Kazhikhov and Smagulov's restriction, $\lambda<2 \mu /(\beta-\alpha)$, should be superfluous. This was shown for $\Omega=\mathbb{R}^{3}$ in [29], by partially following [3], and for the boundary value problem (1) in [4, and by appealing to a quite different method, specific for viscous flows. This approach was applied later on in 30. In [4], the existence of the global regular solution is also proved for small data. In reference [3] the $\lambda^{2}$-terms, which comes out in deducing the full model (see Remark 18 below), are not neglected (see [3], equation $\left.(E)_{1}\right)$. This point was drawn on in [4] and [29]. In [5] the long-time behavior of strong solutions is studied.

In [30, under the assumption that $\lambda / \mu$ is small enough, the existence and uniqueness of global-in-time solutions is shown in two dimensions. Moreover, the convergence, as $\lambda \rightarrow 0$, towards a weak solution of the density-dependent Navier-Stokes problem is established. In the three-dimensional case, global-in-time existence and convergence (as $\lambda \rightarrow 0$ ) towards the dependent-density Navier-Stokes problem is proved in [1], imposing only positive initial density $\left(\rho_{0} \geq 0\right)$.

In [9, the existence and uniqueness of strong solutions are proved by using the semi-Galerkin method for the full model, obtaining the existence and uniqueness of strong solutions, both locally and globally in time at the same level of the classical Navier-Stokes equations. Also, decay results are proved when the external force decays exponentially.

Using an iterative method, in [12, the authors showed the existence and uniqueness of strong solutions. Also error estimates are derived.

Since Galerkin methods are much used in numerical simulations, it is important to derive error estimates for them. Even this case of spectral Galerkin method may be used as a preparation and guide for the more practical finite element Galerkin method. Concerning this, a systematic development of error estimates for the spectral Galerkin method applied to the classical Navier-Stokes equations was given in 23. Applying the same method, error estimates for the nonhomogeneous NavierStokes equations were obtained in [28. These error estimates are local in the sense that they depend on functions that grow exponentially with time. As observed in [15, this is the best one may expect without any assumptions about the stability of the solution being approximated. For the classical Navier-Stokes equations, assuming uniform boundedness in time of the $L^{2}$-norm of the gradient of the velocity and exponential stability in the Dirichlet norm of the solution, optimal uniform-in-time error estimates for the velocity in the Dirichlet norm were derived in [15]. In [28], an optimal uniform-in-time error estimate for the velocity in the $L^{2}$-norm was derived, also for the classical Navier-Stokes equations, assuming exponential stability in the $L^{2}$-norm. Also stated in [28] is a result of uniform-in-time error estimates in the $L^{2}$ norm for the nonhomogeneous Navier-Stokes equations. This last result, however, is not optimal. Moreover, it requires the assumption $\boldsymbol{u} \in L^{\infty}\left(0, T, \boldsymbol{H}^{3}(\Omega)\right)$. As 
pointed out in [16, this assumption is pretty restrictive, since it requires a global compatibility condition on the initial data even for the classical Navier-Stokes equations. In [6], error estimates are derived without explicitly assuming stability, but requiring exponential decay of the external force field. This hypothesis though is very restrictive as well, since gravitational forces do not satisfy it.

In [7] error estimates were derived by assuming that the solution $(\boldsymbol{u}, \rho)$ is $p_{0}$ conditionally asymptotically stable. The number $p_{0}$ is required to satisfy $6 \leq p_{0} \leq$ $\infty$, and is related to the regularity of allowed perturbations of the density equation. In [15], a similar notion was used to treat the classical Navier-Stokes equations (see also [28). The authors adapted it in the proper way to be used in the variable density case. With this assumption, they obtained an optimal uniform-in-time error estimate in the Dirichlet norm for the velocity. An error estimate depending on time for the density in some spaces $L^{r}$ is also derived.

Rummler [24], 25] and 26] has derived a complete description of the eigenfunctions of the Stokes operator in special domains, which could be used to numerically test the results obtained in this work. In fact, these numerical tests are under study and will be cause for a future work.

1.4. Notation and some preliminary results. We collect here as a reference some standard notation used throughout the paper. By $L^{p}(\Omega)$ and $H^{s}(\Omega), 1 \leq p \leq$ $\infty, s=0,1, \ldots$, we denote the classical Lebesgue and Sobolev spaces. The usual norm in $L^{p}(\Omega)$ and $H^{s}(\Omega)$ is indicated by $\|\cdot\|_{L^{p}(\Omega)}$ and $\|\cdot\|_{H^{s}(\Omega)}$, respectively. For $p=2$ we denote $\|\cdot\|_{L^{2}(\Omega)}=\|\cdot\|$ and $(\cdot, \cdot)$ the $L^{2}$ inner product.

We now introduce the standard spaces of the Navier-Stokes framework:

$$
\begin{aligned}
& \boldsymbol{H}=\left\{\boldsymbol{u} \in \boldsymbol{L}^{2}(\Omega): \nabla \cdot \boldsymbol{u}=0, \boldsymbol{u} \cdot \boldsymbol{n}=0 \text { on } \partial \Omega\right\}, \\
& \mathbf{V}=\left\{\boldsymbol{u} \in \boldsymbol{H}^{1}(\Omega): \nabla \cdot \boldsymbol{u}=0, \boldsymbol{u}=0 \text { on } \partial \Omega\right\}, \\
& L_{0}^{2}(\Omega)=\left\{p \in L^{2}(\Omega): \int_{\Omega} p(\boldsymbol{x}) d \boldsymbol{x}=0\right\} .
\end{aligned}
$$

The norms $\|\boldsymbol{u}\|_{H^{1}}$ and $\|\nabla \boldsymbol{u}\|$ are equivalent in $\boldsymbol{V}$, and $\|\boldsymbol{u}\|_{H^{2}}$ and $\|A \boldsymbol{u}\|$ (where $A$ stands for the Stokes operator defined below) are equivalent in $\boldsymbol{H}^{2}(\Omega) \cap \boldsymbol{V}$ 20, 31. On the other hand, the norms $\|p\|_{H^{1}}$ and $\|\nabla p\|_{L^{2}}$ are equivalent in $H^{1}(\Omega) \cap L_{0}^{2}(\Omega)$.

On the other hand, for the density, let us consider the affine space $(k=2,3, \ldots)$ :

$$
H_{N}^{k}(\Omega)=\left\{\rho \in H^{k}(\Omega): \partial_{\boldsymbol{n}} \rho=0 \text { on } \partial \Omega, \int_{\Omega} \rho(\boldsymbol{x}) d \boldsymbol{x}=\int_{\Omega} \rho_{0}(\boldsymbol{x}) d \boldsymbol{x}\right\} .
$$

Obviously, $H_{N}^{k}(\Omega)=\overline{\rho_{0}}+H_{N, 0}^{k}(\Omega)$, where $\overline{\rho_{0}}=\frac{1}{|\Omega|} \int_{\Omega} \rho_{0}(\boldsymbol{x}) d \boldsymbol{x}$, and

$$
H_{N, 0}^{k}(\Omega)=\left\{\rho \in H^{k}(\Omega): \partial_{\boldsymbol{n}} \rho=0 \text { on } \partial \Omega, \int_{\Omega} \rho(\boldsymbol{x}) d \boldsymbol{x}=0\right\} .
$$

Therefore, $H_{N, 0}^{k}(\Omega)$ is a closed subspace of $H_{N}^{k}(\Omega)$. Consequently, thanks to the $H^{2}$ - and $H^{3}$ - regularity of the Poisson-Neumann problem, norms $\|\rho\|_{H^{2}}$ and $\|\Delta \rho\|_{L^{2}}$ are equivalent in $H_{N}^{2}(\Omega)$ and $\|\rho\|_{H^{3}}$ and $\|\nabla \Delta \rho\|$ are equivalent in $H_{N}^{3}(\Omega)$ [4.

Let $-\infty \leq a<b \leq+\infty$ and let $X$ be a Banach space. Then $L^{p}(a, b ; X)$ denotes the space of all measurable-Bochner, $X$-valued functions on $(a, b)$ such that $\int_{a}^{b}\|f(s)\|_{X}^{p} \mathrm{~d} s<\infty$ for $1 \leq p<\infty$ or $\operatorname{ess} \sup _{s \in(a, b)}\|f(s)\|_{X}<\infty$ for $p=\infty$. On the other hand, $L_{l o c}^{p}(a, b ; X)$ contains all the $X$-valued functions defined on $(a, b)$ which belong to $L^{p}(I ; X)$ for any compact subset $I$ of the open set $(a, b)$. By $C^{k}([a, b] ; X)$ 
we denote the space of $X$-valued functions having continuous derivatives less than or equal to $k$ such that $\sup _{t \in[a, b]} \sum_{i=1}^{k}\left\|\frac{d^{i}}{d t} f(t)\right\|_{X}<\infty$. Moreover, $C_{b}^{k}([a, b] ; X)$ is the set of all $X$-valued functions having bounded continuous derivatives less than or equal to $k$.

Let $A: D(A) \subset \boldsymbol{H} \rightarrow \boldsymbol{H}$ be the Stokes operator defined by $A=P(-\Delta)$ with domain $D(A)=\boldsymbol{H}^{2}(\Omega) \cap \boldsymbol{V}$, where $P: \boldsymbol{L}^{2}(\Omega) \rightarrow \boldsymbol{H}$ is the Helmholtz orthogonal projection. The operator $A$ is self-adjoint and positive, and whose inverse operator $A^{-1}$ is continuous from $\boldsymbol{H}$ into $D(A)$. Since the embedding of $H^{1}(\Omega)$ in $L^{2}(\Omega)$ is compact, the embedding of $\boldsymbol{V}$ in $\boldsymbol{H}$ is compact. Thus, $A^{-1}$ is a self-adjoint continuous compact operator in $\boldsymbol{H}$, and by classical spectral theorems there exist a set of eigenvalues $\left\{\lambda_{i}\right\}_{i=1}^{\infty}$ satisfying $A \boldsymbol{a}_{i}=\lambda_{i} \boldsymbol{a}_{i}$ such that $\lambda_{i} \rightarrow \infty$, and a basis of eigenfunctions $\left\{\boldsymbol{a}_{i}\right\}_{i=1}^{\infty}$ which is complete and orthogonal in the spaces $\boldsymbol{H}, \boldsymbol{V}$ and $D(A)$ equipped with the usual inner products $(\cdot, \cdot),(\nabla \cdot, \nabla \cdot)$, and $(A \cdot, A \cdot)$, respectively. See 1 for the regularity results of the Stokes operator.

On the other hand, let $B: H_{N, 0}^{2}(\Omega) \subset L_{0}^{2}(\Omega) \rightarrow L_{0}^{2}(\Omega)$ be the Poisson-Neumann operator defined by $B=-\Delta$. Clearly, $B$ is self-adjoint and positive, and, moreover, $B^{-1}$ is continuous from $L_{0}^{2}(\Omega)$ into $H_{N, 0}^{2}(\Omega)$. Now, due to the fact that the embedding of $H^{1}(\Omega)$ in $L^{2}(\Omega)$ is compact, the embedding of $L_{0}^{2}(\Omega) \cap H^{1}(\Omega)$ in $L_{0}^{2}(\Omega)$ is compact as well. Therefore, $B^{-1}$ is a self-adjoint continuous compact operator in $L_{0}^{2}(\Omega)$. Then there exist a set of the eigenfunctions $\left\{b_{i}\right\}_{i=1}^{\infty}$ and a set of the eigenvalues $\left\{\tau_{i}\right\}_{i=1}^{\infty}$ satisfying $B b_{i}=\tau_{i} b_{i}$ so that $\tau_{i} \rightarrow \infty$, and a basis of eigenfunctions $\left\{b_{i}\right\}_{i=1}^{\infty}$ which is complete and orthogonal in the spaces $L_{0}^{2}(\Omega), L_{0}^{2}(\Omega) \cap H^{1}(\Omega)$, $H_{N, 0}^{2}(\Omega)$ and $H_{N, 0}^{3}(\Omega)$ endowed with the inner products $(\cdot, \cdot),(\nabla \cdot, \nabla \cdot),(\Delta \cdot, \Delta \cdot)$, and $(\nabla \Delta \cdot, \nabla \Delta \cdot)$, respectively. See [21] for the regularity results of the Poisson-Neumann operator.

Let us define $\boldsymbol{V}_{k}=\operatorname{span}\left\{\boldsymbol{a}_{1}, \ldots, \boldsymbol{a}_{k}\right\}$ and $D_{k}=\operatorname{span}\left\{b_{1}, \ldots, b_{k}\right\}$ as the finite vector space spanned by the first $k \in \mathbb{N}$ eigenfunctions associated to the Stokes operator and the Poisson-Neumann problem, respectively. Associated to each finite space, we define $P_{k}: \boldsymbol{H} \rightarrow \boldsymbol{V}_{k}$ and $G_{k}: H^{1}(\Omega) \cap L_{0}^{2}(\Omega) \rightarrow D_{k}$ as the orthogonal projections with respect to $(\cdot, \cdot)$, and $Q_{k}=I-P_{k}$ and $H_{k}=I-G_{k}$.

The following lemma gives us the error estimates for $Q_{k}=I-P_{k}$ and $H_{k}=I-G_{k}$ in several norms. We will use this later to derive long-time error estimates for density and velocity based on constructing Galerkin approximations to $(\boldsymbol{u}, \rho)$ in $\boldsymbol{V}_{k} \times D_{k}$. We refer the reader to [23] for a proof for the Stokes operator being easily extensible for the Poisson-Neumann operator.

Lemma 1. If $(\boldsymbol{u}, \rho) \in \boldsymbol{V} \times L_{0}^{2}(\Omega) \cap H^{1}(\Omega$, then we have the error estimates:

$$
\left\|Q_{k} \boldsymbol{u}\right\|^{2} \leq \frac{1}{\lambda_{k+1}}\|\nabla \boldsymbol{u}\|^{2} \quad \text { and } \quad\left\|H_{k} \rho\right\|^{2} \leq \frac{1}{\tau_{k+1}}\|\nabla \rho\|^{2} .
$$

Moreover, if $(\boldsymbol{u}, \rho) \in D(A) \times H_{N, 0}^{2}(\Omega)$, then

$$
\left\|\nabla Q_{k} \boldsymbol{u}\right\|^{2} \leq \frac{1}{\lambda_{k+1}}\|A \boldsymbol{u}\|^{2} \quad \text { and } \quad\left\|\nabla H_{k} \rho\right\|^{2} \leq \frac{1}{\tau_{k+1}}\|\Delta \rho\|^{2}
$$

and

$$
\left\|Q_{k} \boldsymbol{u}\right\|^{2} \leq \frac{1}{\lambda_{k+1}^{2}}\|A \boldsymbol{u}\|^{2} \quad \text { and } \quad\left\|H_{k} \rho\right\|^{2} \leq \frac{1}{\tau_{k+1}^{2}}\|\Delta \rho\|^{2} \text {. }
$$


Finally, if $\rho \in H_{N, 0}^{3}(\Omega)$, then

$$
\left\|\Delta H_{k} \rho\right\|^{2} \leq \frac{1}{\tau_{k+1}}\|\nabla \Delta \rho\|^{2} .
$$

An easy consequence of the orthogonality of the eigenfunctions is the following stability result.

Lemma 2. If $(\boldsymbol{u}, \rho) \in \boldsymbol{V} \times H^{1}(\Omega) \cap L_{0}^{2}(\Omega)$, then

$$
\begin{aligned}
\left\|\nabla P_{k} \boldsymbol{u}\right\| \leq\|\nabla \boldsymbol{u}\| \quad \text { and } & \left\|\nabla G_{k} \rho\right\| \leq\|\nabla \rho\| . \\
\text { If }(\boldsymbol{u}, \rho) \in \boldsymbol{H}^{2}(\Omega) \cap \boldsymbol{V} \times H_{N, 0}^{2}(\Omega), \text { then } & \\
\left\|A P_{k} \boldsymbol{u}\right\| \leq\|A \boldsymbol{u}\| \quad \text { and } \quad & \left\|\Delta G_{k} \rho\right\| \leq\|\Delta \rho\| .
\end{aligned}
$$

Moreover, if $\rho \in H_{N, 0}^{3}(\Omega)$, then

$$
\left\|\nabla \Delta G_{k} \rho\right\| \leq\|\nabla \Delta \rho\| .
$$

\section{Error estimates for spectral approximation}

Let us introduce here the concept of perturbations of solutions to (11) analogous to [15] and [7. The difference in definition of perturbations among these two works lies in the decay rate as time goes to infinity. To be more precise, in [15], an exponential decay rate is assumed, whereas, in [7, any decay rate is considered.

Definition 3. A pair $(\boldsymbol{\xi}, \eta)$ is called a perturbation of $(\boldsymbol{u}, \rho)$, if $(\widehat{\boldsymbol{u}}=\boldsymbol{u}+\boldsymbol{\xi}, \widehat{\rho}=\rho+\eta)$ is a solution of (11)-3. Then, for a fixed $t_{0} \geq 0,(\boldsymbol{\xi}, \eta)$ is a solution of the initialboundary value problem

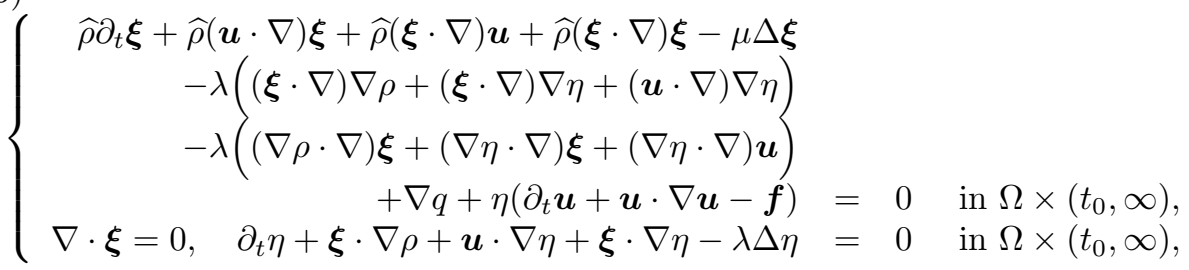

with the boundary conditions

$$
\boldsymbol{\xi}=0, \quad \partial_{\boldsymbol{n}} \eta=0 \text { on } \partial \Omega \times\left(t_{0}, \infty\right),
$$

and the initial conditions

$$
\boldsymbol{\xi}\left(t_{0}\right)=\boldsymbol{\xi}_{0}, \quad \eta\left(t_{0}\right)=\eta_{0} \quad \text { in } \Omega .
$$

Throughout this paper we assume the following hypotheses on the regular solution to be approximated.

(H1) The data satisfy

$\boldsymbol{u}_{0} \in \boldsymbol{V} \cap \boldsymbol{H}^{2}(\Omega), \quad \rho_{0} \in H_{N}^{3}(\Omega), \quad \boldsymbol{f} \in C\left([0, \infty) ; \boldsymbol{H}^{1}(\Omega)\right), \quad \partial_{t} \boldsymbol{f} \in C\left([0, \infty) ; \boldsymbol{L}^{6 / 5}(\Omega)\right)$.

(H2) There exist a global strong solution to (11) such that $(\boldsymbol{u}, \rho) \in C([0,+\infty) ; \boldsymbol{V} \times$ $\left.H_{N}^{2}(\Omega)\right)$ with $\left(\partial_{t} \boldsymbol{u}, \partial_{t} \rho\right) \in L^{2}\left(0,+\infty ; \boldsymbol{L}^{2}(\Omega) \times H^{1}(\Omega)\right)$ and $(\Delta \boldsymbol{u}, \Delta \rho) \in$ $L^{2}\left(0,+\infty ; \boldsymbol{L}^{2}(\Omega) \times H^{1}(\Omega)\right)$, and two constants $M_{1}, M_{2}>0$ such that

$$
\|\nabla \boldsymbol{u}(t)\| \leq M_{1} \text { and }\|\Delta \rho(t)\| \leq M_{2} \text { for all } t \in[0, \infty) .
$$


(H3) Suppose that $(\boldsymbol{u}, \rho)$ is conditionally asymptotically stable, that is, there exist positive numbers $\delta_{1}, \delta_{2}, A_{1}, A_{2}$ and two continuous decreasing functions $F, G:[0, \infty) \rightarrow \mathbb{R}^{+}$such that $G(0)=F(0)=1$, and $\lim _{t \rightarrow \infty} F(t)=$ $\lim _{t \rightarrow \infty} G(t)=0$ so that for every $t_{0} \geq 0$ and for every $\boldsymbol{\xi}_{0} \in \boldsymbol{V} \cap \boldsymbol{H}^{2}(\Omega)$ and $\eta_{0} \in H^{3}(\Omega)$ with $\partial_{\boldsymbol{n}} \eta_{0}=0$ on $\Sigma$, satisfying $\left\|\nabla \boldsymbol{\xi}_{0}\right\|<\delta_{1}$ and $\left\|\Delta \eta_{0}\right\|<\delta_{2}$, problem (5) is uniquely solvable with

$$
\begin{gathered}
\boldsymbol{\xi} \in L_{l o c}^{2}\left(\left[t_{0}, \infty\right) ; \boldsymbol{V} \cap \boldsymbol{H}^{2}(\Omega)\right), \\
\partial_{t} \boldsymbol{\xi} \in L_{l o c}^{2}\left(\left[t_{0}, \infty\right) ; \boldsymbol{H}^{1}(\Omega)\right), \\
\eta \in L_{l o c}^{2}\left(\left[t_{0}, \infty\right) ; H_{N, 0}^{3}(\Omega)\right), \\
\partial_{t} \eta \in L_{l o c}^{2}\left(\left[t_{0}, \infty\right) ; H_{N, 0}^{2}(\Omega)\right) .
\end{gathered}
$$

Moreover,

$$
\begin{aligned}
\|\nabla \boldsymbol{\xi}(t)\| \leq A_{1}\left\|\nabla \boldsymbol{\xi}_{0}\right\| F\left(t-t_{0}\right) & \forall t \geq t_{0} \\
\|\Delta \eta(t)\| \leq A_{2}\left\|\Delta \eta_{0}\right\| G\left(t-t_{0}\right) & \forall t \geq t_{0} .
\end{aligned}
$$

Remark 4. Hypothesis (H2) is established for two-dimensional domains under no restrictions on hypothesis ( $H 1)$, but as usual, for three-dimensional domains, some kind of smallness on the norms $\left\|\boldsymbol{u}_{0}\right\|_{H^{1}(\Omega)},\left\|\rho_{0}\right\|_{H^{2}(\Omega)}$ and $\|\boldsymbol{f}\|_{L^{\infty}\left(0, \infty ; \boldsymbol{L}^{2}(\Omega)\right)}$ referred to in hypothesis (H1) must be imposed. On the other hand, a regularization argument shows that one may assume that $\boldsymbol{f} \in L^{\infty}\left(0, \infty ; \boldsymbol{H}^{1}(\Omega)\right)$ and $\partial_{t} \boldsymbol{f} \in L^{\infty}\left(0, \infty ; \boldsymbol{L}^{6 / 5}(\Omega)\right)$ as in the case of the Navier-Stokes equations [16].

Remark 5. The existence of perturbations, that is, hypothesis (H3) is true, is left hanging for the moment until obtaining better estimates for the exact solution $(\boldsymbol{u}, \rho)$ based on $(H 1)$ and $(H 2)$. An important observation is that our definition of perturbations is slightly different from that of Heywood and Rannacher for the Navier-Stokes equations since the perturbed solution, $\widehat{\rho}$, appears explicitly in some terms of (5) given that $\widehat{\rho}$ will play an essential role in the following arguments.

It is not hard to prove from the continuum equation (11) 3 that $\int_{\Omega} \rho(t, \boldsymbol{x}) d \boldsymbol{x}=$ $\int_{\Omega} \rho_{0}(\boldsymbol{x}) d \boldsymbol{x}$ for all $t \geq 0$; therefore, the space $D_{k}$ may seem to be somewhat inappropriate to approximate $\rho$ since it is made up of zero mean-value functions. To avoid this inconvenience, as was done in many previous papers in the references below, one observes that $\widetilde{\rho}:=\rho-\overline{\rho_{0}}$ satisfies the same equation as $\rho$. Then the spectral Galerkin approximations for $(\boldsymbol{u}, \rho)$ are defined for each $k \in \mathbb{N}$ as the solution $\left(\boldsymbol{u}^{k}, \rho^{k}\right) \in C^{2}\left([0, \infty) ; \boldsymbol{V}_{k}\right) \times C^{2}\left([0, \infty) ; D_{k}\right)$ of

(6)

$$
\begin{gathered}
\left\{\begin{array}{c}
\left(\rho^{k} \partial_{t} \boldsymbol{u}^{k}, \phi^{k}\right)+\left(\left(\rho^{k} \boldsymbol{u}^{k} \cdot \nabla\right) \boldsymbol{u}^{k}, \boldsymbol{\phi}^{k}\right)+\mu\left(A \boldsymbol{u}^{k}, \boldsymbol{\phi}^{k}\right)-\lambda\left(\left(\boldsymbol{u}^{k} \cdot \nabla\right) \nabla \widetilde{\rho}^{k}, \boldsymbol{\phi}^{k}\right) \\
-\lambda\left(\left(\nabla \widetilde{\rho}^{k} \cdot \nabla\right) \boldsymbol{u}^{k}, \boldsymbol{\phi}^{k}\right)=\left(\rho^{k} \boldsymbol{f}, \boldsymbol{\phi}^{k}\right) \quad \forall \boldsymbol{\phi}^{k} \in \boldsymbol{V}_{k},
\end{array}\right. \\
\left(\partial_{t} \widetilde{\rho}^{k}, \nu^{k}\right)+\left(\boldsymbol{u}^{k} \cdot \nabla \widetilde{\rho}^{k}, \nu^{k}\right)-\lambda\left(\Delta \widetilde{\rho}^{k}, \nu^{k}\right)=0 \quad \forall \nu^{k} \in D_{k},
\end{gathered}
$$

where $\rho^{k}=\widetilde{\rho}^{k}+\overline{\rho_{0}}$, and with the initial conditions

$$
\boldsymbol{u}^{k}(\boldsymbol{x}, 0)=P_{k} \boldsymbol{u}_{0} \text { and } \widetilde{\rho}^{k}(0, \boldsymbol{x})=G_{k} \widetilde{\rho}_{0}(\boldsymbol{x}),
$$

respectively. Clearly, $\rho^{k}$ satisfies equation (7). The details of existence of approximate solutions to (6)-(7) will be given in the appendix. 
Theorem 6. Under hypotheses (H1), (H2), (H3), there exist a constant $L>0$ and a nonnegative integer $k \in \mathbb{N}$, depending on the problem data, such that

$$
\left\|\nabla\left(\boldsymbol{u}^{k}-\boldsymbol{u}\right)\right\|^{2}+\left\|\Delta\left(\rho^{k}-\rho\right)\right\|^{2} \leq L\left(\frac{1}{\lambda_{k+1}}+\frac{1}{\tau_{k+1}}\right),
$$

for all $t \geq 0$ and $k \geq N$.

\section{Proof of Theorem 6}

The first result concerns better estimates for the solution $(\boldsymbol{u}, \rho)$ to (1) under hypothesis (H2) with no extra requirements on hypothesis $(H 1)$. We include a sketch of its proof for the sake of completeness and to make the reader see that those estimates are reasonable to be expected. A rigorous justification follows by means of semi-Galerkin approximations, for instance, on which we may assume the needed regularity in order to justify any expression in the following lemma. Finally, a uniqueness argument shows that the solution constructed by the semi-Garlekin approach coincides with the solution given in hypothesis (H2). Details can be found in $[9$.

Lemma 7. Under the assumptions (H1) and (H2), the solution $(\boldsymbol{u}, \rho)$ to (1) satisfies the following estimates:

$$
\begin{gathered}
\sup _{t \geq 0}\|A \boldsymbol{u}(t)\|<\infty, \quad \sup _{t \geq 0}\left\|\partial_{t} \boldsymbol{u}(t)\right\|<\infty, \quad \sup _{t \geq 0}\left\|\nabla \partial_{t} \rho(t)\right\|<\infty, \quad \sup _{t \geq 0}\|\nabla \Delta \rho(t)\|<\infty, \\
\sup _{t \geq 0} e^{-\gamma t} \int_{0}^{t} e^{\gamma \tau}\left\|\nabla \partial_{t} \boldsymbol{u}(\tau)\right\|^{2} d \tau<\infty, \quad \sup _{t \geq 0} e^{-\gamma t} \int_{0}^{t} e^{\gamma \tau}\left\|\Delta \partial_{t} \rho(\tau)\right\|^{2} d \tau<\infty,
\end{gathered}
$$

for any $\gamma>0$.

Proof. First, one states a maximum principle for the density

$$
0<\alpha \leq \rho(t, \boldsymbol{x}) \leq \beta<\infty \text { in } \Sigma .
$$

We now use the inequality

(8)

$$
\begin{aligned}
& \frac{d}{d t}\left\{\frac{\mu}{2}\|\nabla \boldsymbol{u}\|^{2}+\lambda\|\Delta \rho\|^{2}\right\}+\frac{m}{2}\left\|\boldsymbol{u}_{t}\right\|^{2}+\left\|\nabla \rho_{t}\right\|^{2}+\frac{m \mu^{2}}{16 M^{2}}\|A \boldsymbol{u}\|^{2}+\frac{\lambda^{2}}{4}\|\nabla \Delta \rho\|^{2} \\
& \quad \leq C\left(\|\Delta \rho\|^{2}+\|\nabla \boldsymbol{u}\|^{2}\right)^{3}+C\|\boldsymbol{f}\|^{2},
\end{aligned}
$$

derived in [5] on p. 1232 and in [9] on p. 70. Multiplying (8) by $e^{\gamma t}$, yields

$$
\begin{gathered}
\frac{\mu}{2}\|\nabla \boldsymbol{u}\|^{2}+\lambda\|\Delta \rho\|^{2}+e^{-\gamma t} \int_{0}^{t} e^{\gamma \tau}\left(\frac{m}{2}\left\|\partial_{t} \boldsymbol{u}(\tau)\right\|^{2}+\left\|\partial_{t} \nabla \rho(\tau)\right\|^{2}\right) d \tau \\
+e^{-\gamma t} \int_{0}^{t} e^{\gamma \tau}\left(\frac{m \mu^{2}}{16 M^{2}}\|A \boldsymbol{u}(\tau)\|^{2}+\frac{\lambda^{2}}{4}\|\nabla \Delta \rho(\tau)\|^{2}\right) d \tau \leq C
\end{gathered}
$$

where we have used hypotheses $(H 1)$ and $(H 2)$. Next, the stronger bounds for the velocity time derivative are accomplished by differentiating equation (1) with respect to time and taking the $L^{2}$-inner product of $\partial_{t} \boldsymbol{u}$ with the resulting equation, and for the density time derivatives are accomplished by differentiating equation 
(11) 2 with respect to time and taking the $L^{2}(\Omega)$-inner product of $-\Delta \partial_{t} \rho$ with the resulting equation. Thus, one gets

$$
\begin{aligned}
& \frac{d}{d t}\left(\left\|\rho^{1 / 2} \partial_{t} \boldsymbol{u}\right\|^{2}+\left\|\nabla \partial_{t} \rho\right\|^{2}\right)+\mu\left\|\nabla \partial_{t} \boldsymbol{u}\right\|^{2}+\lambda\left\|\Delta \partial_{t} \rho\right\|^{2} \\
& \leq C\left(1+\|\boldsymbol{f}\|^{2}\right)\left\|\nabla \partial_{t} \rho\right\|^{2}+C\left\|\partial_{t} \boldsymbol{u}\right\|^{2}+C\left\|\partial_{t} \boldsymbol{f}\right\|_{L^{6 / 5}(\Omega)}^{2} .
\end{aligned}
$$

Next, multiplying again by $e^{\gamma t}$ and taking into account estimate (9) and hypothesis $(H 1)$, one finds the desired bounds for the time derivatives (see [16, 15, 9]). The remainder of bounds are a consequence of the following estimate:

$$
\mu\|A \boldsymbol{u}\|^{2}+\lambda\|\nabla \Delta \rho\|^{2} \leq C\left\|\partial_{t} \boldsymbol{u}\right\|^{2}+C\left\|\nabla \partial_{t} \rho\right\|^{2}+C\left(\|\nabla \boldsymbol{u}\|^{2}+\|\Delta \rho\|^{2}\right)^{3} .
$$

An obvious consequence of Lemma 7 is the following.

Corollary 8. For all $t_{0}, t$ with $0 \leq t_{0} \leq t$, we have

$$
\int_{t_{0}}^{t}\left(\left\|\nabla \partial_{t} \boldsymbol{u}(\tau)\right\|^{2}+\left\|\Delta \partial_{t} \rho(\tau)\right\|^{2}\right) d \tau \leq C\left(t-t_{0}\right)+C .
$$

Proof. The proof follows from (10).

Remark 9. We may have assumed Lemma 7 as a hypothesis, but it is not really necessary since it is a consequence of hypothesis $(H 1)$ and $(H 2)$. Therefore, we prefer assuming hypothesis $(H 2)$ to imposing smallness on hypothesis $(H 1)$ in three dimensions. Moreover, we can also expect that $\left(\sigma \partial_{t t} \boldsymbol{u}, \partial_{t t} \rho\right) \in L_{l o c}^{2}\left(0,+\infty ; \boldsymbol{L}^{2}(\Omega) \times\right.$ $\left.H^{1}(\Omega)\right)$ with $\sigma(t)=\min \{t, 1\}$ (see $[9]$ ).

It is at this point where we can justify, before proceeding any further, that we have not just introduced a vacuous definition of perturbations. We follow the ideas of Heywood and Rannacher in [17. Let $(\widehat{\boldsymbol{u}}, \widehat{\rho})$ be a perturbed solution of (1) such that

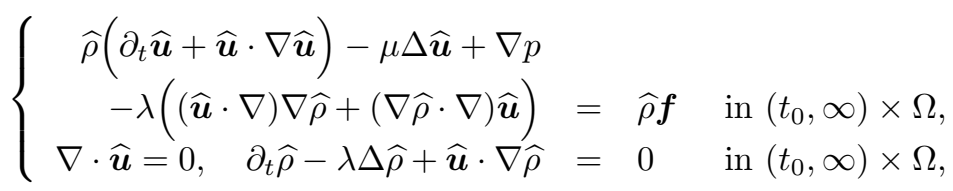

supplemented by the boundary conditions

$$
\widehat{\boldsymbol{u}}=0, \quad \partial_{\boldsymbol{n}} \widehat{\rho}=0 \text { on } \Sigma,
$$

and the initial conditions

$$
\widehat{\boldsymbol{u}}\left(t_{0}\right)=\widehat{\boldsymbol{u}}_{0}, \quad \widehat{\rho}\left(t_{0}\right)=\widehat{\rho}_{0} \text { in } \Omega,
$$

with $\left(\widehat{\boldsymbol{u}}_{0}, \widehat{\rho}_{0}\right) \in \boldsymbol{V} \cap \boldsymbol{H}^{2}(\Omega) \times H_{N}^{3}(\Omega)$ being close to $\left(\boldsymbol{u}\left(t_{0}\right), \rho\left(t_{0}\right)\right)$ with $0<\widetilde{\alpha} \leq$ $\widehat{\rho}_{0}(\boldsymbol{x}) \leq \widetilde{\beta}<\infty$ for all $\boldsymbol{x} \in \Omega$. As a perturbation of $(\boldsymbol{u}, \rho)$ we define $(\boldsymbol{\xi}, \eta)$ to be $(\widehat{\boldsymbol{u}}-\boldsymbol{u}, \widehat{\rho}-\rho)$ with $\left(\boldsymbol{\xi}_{0}, \eta_{0}\right)$ being $\left(\widehat{\boldsymbol{u}}_{0}-\boldsymbol{u}\left(t_{0}\right), \widehat{\rho}_{0}-\rho\left(t_{0}\right)\right) \in \boldsymbol{V} \cap \boldsymbol{H}^{2}(\Omega) \times H_{N, 0}^{3}(\Omega)$. Clearly, $(\boldsymbol{\xi}, \eta)$ satisfy (5), at least formally. With this definition of perturbations we can only guarantee the local strong existence for (5) since it is related to the existence of strong perturbed solutions that is at first ensured in a local time interval by the well-known local existence theorem for (1) in three dimensions (being global in two dimensions) described in the introduction. Therefore, one may assure the existence of some $T^{*}>t_{0}$ and of $(\boldsymbol{\xi}, \eta)$ in the condition of Lemma 7 and Remark 9. That is, $(\boldsymbol{\xi}, \eta) \in C\left(\left[t_{0}, T^{*}\right) ; \boldsymbol{V} \cap H^{2}(\Omega) \times H_{N}^{3}(\Omega)\right) \cap C^{1}\left(\left[t_{0}, T^{*}\right) ; L^{2}(\Omega) \times H^{1}(\Omega)\right)$ with $\left(\partial_{t t} \boldsymbol{\xi}, \partial_{t t} \eta\right) \in L^{2}\left(t_{0}, T^{*} ; \boldsymbol{L}^{2}(\Omega) \times \boldsymbol{H}^{1}(\Omega)\right)$ satisfying system (5) (see [14, 16, 9]). 
The following result shows that such a local existence time interval for perturbations becomes also global in three dimensions.

Lemma 10. Suppose that conditions (H1) and (H2) are satisfied. Then there exist positive numbers $\delta, A, B$ so that for every $t_{0} \geq 0$ and for every $\boldsymbol{\xi}_{0} \in \boldsymbol{V} \cap \boldsymbol{H}^{2}(\Omega)$ and $\eta_{0} \in H_{N, 0}^{3}(\Omega)$, satisfying $\left\|\nabla \boldsymbol{\xi}_{0}\right\|^{2}+\left\|\Delta \eta_{0}\right\|^{2}<\delta$, problem (5) is uniquely solvable with

$$
\begin{gathered}
\boldsymbol{\xi} \in L_{l o c}^{2}\left(\left[t_{0}, \infty\right) ; \boldsymbol{V} \cap \boldsymbol{H}^{2}(\Omega)\right), \\
\partial_{t} \boldsymbol{\xi} \in L_{l o c}^{2}\left(\left[t_{0}, \infty\right) ; \boldsymbol{H}^{1}(\Omega)\right), \\
\eta \in L_{l o c}^{2}\left(\left[t_{0}, \infty\right) ; H^{3}(\Omega)\right), \\
\partial_{t} \eta \in L_{l o c}^{2}\left(\left[t_{0}, \infty\right) ; H^{2}(\Omega)\right) .
\end{gathered}
$$

Moreover,

$$
\|\nabla \boldsymbol{\xi}(t)\|^{2}+\|\Delta \eta(t)\|^{2} \leq A\left(\left\|\nabla \boldsymbol{\xi}_{0}\right\|^{2}+\left\|\Delta \eta_{0}\right\|^{2}\right) e^{-B\left(t-t_{0}\right)} \quad \forall t \geq t_{0}
$$

Proof. As indicated above, suppose that $(\boldsymbol{\xi}, \eta)$ is in the condition of Lemma 7 and Remark 9 so that system (5) holds in $\left(t_{0}, T^{*}\right) \times \Omega$ for some $T^{*}>0$. Let us now show that $T^{*}=+\infty$. We can admit that $0<\widetilde{\alpha} \leq \widehat{\rho}(\boldsymbol{x}, t) \leq \widetilde{\beta}<\infty$ holds in $(t, \boldsymbol{x}) \in\left[t_{0}, T^{*}\right) \times \Omega$ by maximum principle. Setting as a test function, $\partial_{t} \boldsymbol{u}$ into the variational form of the perturbation equation (5) 1 gives

$$
\begin{aligned}
&\left\|\hat{\rho}^{1 / 2} \partial_{t} \boldsymbol{\xi}\right\|^{2}+\frac{d}{d t} \frac{\mu}{2}\|\nabla \boldsymbol{\xi}\|^{2} \\
&=-\left(\widehat{\rho}(\boldsymbol{u} \cdot \nabla) \boldsymbol{\xi}, \partial_{t} \boldsymbol{\xi}\right)-\left(\widehat{\rho}(\boldsymbol{\xi} \cdot \nabla) \boldsymbol{u}, \partial_{t} \boldsymbol{\xi}\right)-\left(\widehat{\rho}(\boldsymbol{\xi} \cdot \nabla) \boldsymbol{\xi}, \partial_{t} \boldsymbol{\xi}\right) \\
&+\lambda\left((\boldsymbol{\xi} \cdot \nabla) \nabla \rho, \partial_{t} \boldsymbol{\xi}\right)+\lambda\left((\boldsymbol{\xi} \cdot \nabla) \nabla \eta, \partial_{t} \boldsymbol{\xi}\right)+\lambda\left((\boldsymbol{u} \cdot \nabla) \nabla \eta, \partial_{t} \boldsymbol{\xi}\right) \\
&+\lambda\left((\nabla \rho \cdot \nabla) \boldsymbol{\xi}, \partial_{t} \boldsymbol{\xi}\right)+\lambda\left((\nabla \eta \cdot \nabla) \boldsymbol{\xi}, \partial_{t} \boldsymbol{\xi}\right)+\lambda\left((\nabla \eta \cdot \nabla) \boldsymbol{u}, \partial_{t} \boldsymbol{\xi}\right) \\
&-\left(\eta\left(\partial_{t} \boldsymbol{u}+\boldsymbol{u} \cdot \nabla \boldsymbol{u}-\boldsymbol{f}\right), \partial_{t} \boldsymbol{\xi}\right) .
\end{aligned}
$$

In estimating the right-hand side of (12) we use Sobolev's and Hölder's inequality, and Lemma 7 to get

$$
\begin{aligned}
\frac{\mu}{2} \frac{d}{d t}\|\nabla \boldsymbol{\xi}\|^{2}+\frac{3 \widetilde{\alpha}}{4}\left\|\partial_{t} \boldsymbol{\xi}\right\|^{2} \leq & C\left(\|\nabla \boldsymbol{\xi}\|^{2}+\|\Delta \eta\|^{2}\right)^{3} \\
& +\delta\|A \boldsymbol{\xi}\|^{2}+\gamma\|\nabla \Delta \eta\|^{2},
\end{aligned}
$$

with $\gamma$ and $\delta$ being constants from Young's inequality. Next, we test (5) with $\boldsymbol{v}=A \boldsymbol{\xi}$, yielding

$$
\begin{aligned}
\mu\|A \boldsymbol{\xi}\|^{2}= & -(\widehat{\rho}(\boldsymbol{u} \cdot \nabla) \boldsymbol{\xi}, A \boldsymbol{\xi})-(\widehat{\rho}(\boldsymbol{\xi} \cdot \nabla) \boldsymbol{u}, A \boldsymbol{\xi})-(\widehat{\rho}(\boldsymbol{\xi} \cdot \nabla) \boldsymbol{\xi}, A \boldsymbol{\xi}) \\
& +\lambda((\boldsymbol{\xi} \cdot \nabla) \nabla \rho, A \boldsymbol{\xi})+\lambda((\boldsymbol{\xi} \cdot \nabla) \nabla \eta, A \boldsymbol{\xi})+\lambda((\boldsymbol{u} \cdot \nabla) \nabla \eta, A \boldsymbol{\xi}) \\
& +\lambda((\nabla \rho \cdot \nabla) \boldsymbol{\xi}, A \boldsymbol{\xi})+\lambda((\nabla \eta \cdot \nabla) \boldsymbol{\xi}, A \boldsymbol{\xi})+\lambda((\nabla \eta \cdot \nabla) \boldsymbol{u}, A \boldsymbol{\xi}) \\
& -\left(\widehat{\rho} \partial_{t} \boldsymbol{\xi}, A \boldsymbol{\xi}\right)-\left(\eta\left(\partial_{t} \boldsymbol{u}+\boldsymbol{u} \cdot \nabla \boldsymbol{u}-\boldsymbol{f}\right), A \boldsymbol{\xi}\right) .
\end{aligned}
$$

The terms on the right-hand side are handled exactly in the same way as before. We only focus on the term

$$
\left|\left(\widetilde{\rho} \partial_{t} \boldsymbol{\xi}, A \boldsymbol{\xi}\right)\right| \leq \widetilde{\beta}\left\|\partial_{t} \boldsymbol{\xi}\right\|\|A \boldsymbol{\xi}\| \leq \frac{\widetilde{\beta}^{2}}{\mu}\left\|\partial_{t} \boldsymbol{\xi}\right\|^{2}+\frac{\mu}{4}\|A \boldsymbol{\xi}\|^{2} .
$$


Thus, one gets

$$
\frac{\mu}{2}\|A \boldsymbol{\xi}\|^{2} \leq \frac{\widetilde{\beta}^{2}}{\mu}\left\|\partial_{t} \boldsymbol{\xi}\right\|^{2}+C\left(\|\nabla \boldsymbol{\xi}\|^{2}+\|\Delta \eta\|^{2}\right)^{3}+\gamma\|\nabla \Delta \eta\|^{2} .
$$

Multiplying (15) by $\frac{\widetilde{\alpha} \mu}{4 \widetilde{\beta}^{2}}$ and adding to (13) yields

$$
\begin{aligned}
\frac{\mu}{2} \frac{d}{d t} \| & \nabla \boldsymbol{\xi}\left\|^{2}+\frac{\widetilde{\alpha}}{2}\right\| \partial_{t} \boldsymbol{\xi}\left\|^{2}+\frac{\widetilde{\alpha} \mu^{2}}{8 \widetilde{\beta}^{2}}\right\| A \boldsymbol{\xi} \|^{2} \\
& \leq C\left(\|\Delta \eta\|^{2}+\|\nabla \boldsymbol{\xi}\|^{2}\right)^{3}+\delta\|A \boldsymbol{\xi}\|^{2}+\gamma\|\nabla \Delta \eta\|^{2} .
\end{aligned}
$$

On the other hand, multiplying (5) $)_{3}$ by $-\Delta \partial_{t} \eta+\lambda \Delta^{2} \eta$ and integrating by parts, one obtains

$\left\|\nabla \partial_{t} \eta\right\|^{2}+\frac{\lambda}{2} \frac{d}{d t}\|\Delta \eta\|^{2}+\lambda^{2}\|\nabla \Delta \eta\|^{2}=\left(\nabla(\boldsymbol{\xi} \cdot \nabla \rho+\boldsymbol{u} \cdot \nabla \eta+\boldsymbol{\xi} \cdot \nabla \eta),-\nabla \partial_{t} \eta+\nabla \Delta \eta\right)$.

Using Hölder's and Sobolev's inequality in the previous equality, we have (for an arbitrary $\delta>0$ )

$$
\frac{\lambda}{2} \frac{d}{d t}\|\Delta \eta\|^{2}+\left\|\nabla \partial_{t} \eta\right\|^{2}+\frac{\lambda^{2}}{2}\|\nabla \Delta \eta\|^{2} \leq C\left(\|\nabla \boldsymbol{\xi}\|^{2}+\|\Delta \eta\|^{2}\right)^{3}+\delta\|A \boldsymbol{\xi}\|^{2} .
$$

Finally, if we add inequalities (17) and (18) and choose $\gamma$ and $\delta$ small enough, we obtain

$$
\begin{aligned}
\frac{d}{d t}\{ & \left.\frac{\mu}{2}\|\nabla \boldsymbol{\xi}\|^{2}+\frac{\lambda}{2}\|\Delta \eta\|^{2}\right\}+\frac{\widetilde{\alpha}}{2}\left\|\partial_{t} \boldsymbol{\xi}\right\|^{2}+\left\|\nabla \partial_{t} \eta\right\|^{2} \\
& +\frac{\widetilde{\alpha} \mu^{2}}{16 \widetilde{\beta}^{2}}\|A \boldsymbol{\xi}\|^{2}+\frac{\lambda^{2}}{4}\|\nabla \Delta \eta\|^{2} \leq C\left(\|\nabla \boldsymbol{\xi}\|^{2}+\|\Delta \eta\|^{2}\right)^{3} .
\end{aligned}
$$

Next, examining the proof of global existence for small data and $f=0$ given in 4], one may see that there exists a small $\delta>0$ such that if $\left\|\nabla \boldsymbol{\xi}_{0}\right\|^{2}+\left\|\Delta \eta_{0}\right\|^{2}<\delta$, then it holds that

$$
\frac{\mu}{2}\|\nabla \boldsymbol{\xi}(t)\|^{2}+\frac{\lambda}{2}\|\Delta \eta(t)\|^{2} \leq\left(\frac{\mu}{2}\left\|\nabla \boldsymbol{\xi}_{0}\right\|^{2}+\frac{\lambda}{2}\left\|\Delta \eta_{0}\right\|^{2}\right) e^{-C\left(t-t_{0}\right)}
$$

and

$$
e^{-\gamma t} \int_{t_{0}}^{t} e^{\gamma \tau}\left(\frac{\widetilde{\alpha}}{2}\left\|\partial_{t} \boldsymbol{\xi}(s)\right\|^{2}+\left\|\nabla \partial_{t} \eta(s)\right\|^{2}+\frac{\widetilde{\alpha} \mu^{2}}{16 \widetilde{\beta}^{2}}\|A \boldsymbol{\xi}(s)\|^{2}+\frac{\lambda}{4}\|\nabla \Delta \eta(s)\|^{2}\right) d s \leq C .
$$

From this last result it is easy to complete the proof.

Remark 11. Note that hypothesis (H3) is more general than Lemma10 in the sense that perturbations are allowed to decay in any way as time goes towards infinity and the initial data $\left(\boldsymbol{\xi}_{0}, \eta_{0}\right)$ are assumed to be small independently.

Remark 12. In [18, several interesting notions of stability are treated for the NavierStokes equations. We plan to extend that analysis to the model studied in this work.

The next result is related to a priori estimates for perturbations $(\boldsymbol{\xi}, \eta)$ satisfying (51) analogous to those in Lemma 7. To accomplish this, let $\left(\boldsymbol{\xi}_{0}, \eta_{0}\right)$ be such that $\left\|\nabla \boldsymbol{\xi}_{0}\right\|<\delta_{1}$ and $\left\|\Delta \eta_{0}\right\|<\delta_{2}$ as in hypothesis (H3), so $\|\nabla \boldsymbol{\xi}(t)\| \leq A_{1} \delta_{1}$ and $\|\Delta \eta(t)\| \leq A_{2} \delta_{2}$ for all $t \geq t_{0}$. Thus, one finds that $(\widehat{\boldsymbol{u}}=\boldsymbol{u}+\boldsymbol{\xi}, \widehat{\rho}=\rho+\eta)$ being a solution to (1) satisfies $\|\nabla \widehat{\boldsymbol{u}}(t)\| \leq M_{1}+A_{1} \delta_{1}$ and $\|\Delta \widehat{\rho}(t)\| \leq M_{2}+A_{2} \delta_{2}$ for all 
$t \geq t_{0}$. Then one can readily see that $(\widehat{\boldsymbol{u}}, \widehat{\rho})$ holds for Lemma 7 . Therefore, $(\boldsymbol{\xi}, \eta)$ inherits from $(\boldsymbol{u}, \rho)$ the following result.

Lemma 13. Under the assumptions $(H 1),(H 2)$, and $(H 3)$, the solution $(\boldsymbol{\xi}, \eta)$ to (5) satisfies

$$
\begin{gathered}
\sup _{t \geq t_{0}}\|A \boldsymbol{\xi}(t)\|<\infty, \quad \sup _{t \geq t_{0}}\left\|\partial_{t} \boldsymbol{\xi}(t)\right\|<\infty, \quad \sup _{t \geq t_{0}}\left\|\nabla \partial_{t} \eta(t)\right\|<\infty, \quad \sup _{t \geq t_{0}}\|\nabla \Delta \eta(t)\|<\infty, \\
\quad \sup _{t \geq t_{0}} e^{-\gamma t} \int_{0}^{t} e^{\gamma \tau}\left\|\nabla \partial_{t} \boldsymbol{\xi}(\tau)\right\|^{2} d \tau<\infty, \quad \sup _{t \geq t_{0}} e^{-\gamma t} \int_{0}^{t} e^{\gamma \tau}\left\|\Delta \partial_{t} \eta(\tau)\right\|^{2} d \tau<\infty
\end{gathered}
$$

for any $\gamma>0$.

Corollary 14. For all $t_{0}, t$ with $0 \leq t_{0} \leq t$, we have

$$
\int_{t_{0}}^{t}\left\|\nabla \partial_{t} \boldsymbol{\xi}(\tau)\right\|^{2} d \tau \leq C\left(t-t_{0}\right)+C .
$$

For future reference, we let $\boldsymbol{u}=\sum_{n=1}^{\infty} c_{n}(t) \boldsymbol{a}_{n}(\boldsymbol{x})$ and $\widetilde{\rho}=\sum_{n=1}^{\infty} d_{n}(t) b_{n}(\boldsymbol{x})$ be the series with respect to the eigenfunctions of the solution $(\boldsymbol{u}, \rho)$ of problem (11). Associated to these two series, we define $\boldsymbol{v}^{k}=\sum_{n=1}^{k} c_{n}(t) \boldsymbol{a}^{n}(\boldsymbol{x})$ and $\delta^{k}=$ $\sum_{n=1}^{k} d_{n}(t) b^{n}(\boldsymbol{x})$ to be the $k$ th partial sums of the series for $(\boldsymbol{u}, \rho)$. This truncation series may be seen as an orthogonal projection onto $\boldsymbol{V}_{k}$ and $D_{k}$ with respect to the $L^{2}$-inner product.

Let $\boldsymbol{e}_{\boldsymbol{u}}^{k}=\boldsymbol{u}-\boldsymbol{v}^{k}$, and let $\boldsymbol{w}^{k}=\boldsymbol{u}^{k}-\boldsymbol{v}^{k}$, where $\boldsymbol{u}^{k}$ is the $k$ th Galerkin approximation. Then $\boldsymbol{u}-\boldsymbol{u}^{k}=\boldsymbol{e}_{\boldsymbol{u}}^{k}-\boldsymbol{w}^{k}$. Analogously, for the density, we denote by $\psi^{k}=\widetilde{\rho}^{k}-\delta^{k}$ and $e_{\rho}^{k}=\widetilde{\rho}-\delta^{k}$, hence $\rho-\rho^{k}=\widetilde{\rho}-\widetilde{\rho}^{k}=e_{\rho}^{k}-\psi^{k}$. Clearly, in order to prove that $\left\|\nabla\left(\boldsymbol{u}^{k}-\boldsymbol{u}\right)\right\|+\left\|\Delta\left(\rho^{k}-\rho\right)\right\| \leq L\left(\frac{1}{\lambda_{k+1}}+\frac{1}{\tau_{k+1}}\right)$ on $[0, \infty)$ for some constants $L>0$, it remains to estimate $\boldsymbol{w}^{k}$ and $\psi^{k}$.

To easily understand the proof we split the main result into several parts: Lemmas 15, 16, and 17. After these results we will prove Theorem 6]

One of the main ideas underlying this paper is to establish an approximate maximum principle for $\rho^{k}$. Such a maximum principle is not deduced directly from (77), it is based on the error for $\rho^{k}-\rho$ in the $H^{2}$-norm and the Sobolev embedding $H_{N, 0}^{2}(\Omega) \hookrightarrow L^{\infty}(\Omega)$, say $\|\rho\|_{L^{\infty}(\Omega)} \leq C_{\Omega}\|\Delta \rho\|$. It is important to keep in mind the constant $C_{\Omega}>0$ because it is going to appear several times throughout this work.

Lemma 15. Suppose that there exist a constant $L>0$ and a nonnegative integer $k \in \mathbb{N}$ depending on the problem data in such a way that $\left\|\Delta\left(\rho^{k}-\rho\right)\right\|^{2} \leq$ $L\left(\frac{1}{\lambda_{k+1}}+\frac{1}{\tau_{k+1}}\right)$ holds on some interval $\left[0, t^{*}\right]$, for $C_{\Omega}^{2} L\left(\frac{1}{\lambda_{k+1}}+\frac{1}{\tau_{k+1}}\right)<\frac{\alpha^{2}}{4}$. Then $\rho^{k}$ satisfies an approximate maximum principle

$$
0<\frac{\alpha}{2} \leq \rho^{k}(\boldsymbol{x}, t) \leq \widetilde{\beta} \text { in } \Omega \times\left[0, t^{*}\right],
$$

where $\widetilde{\beta}>0$ is related to $\beta$.

Proof. Clearly, one sees that $\rho^{k}-\rho \in H_{N, 0}^{2}(\Omega)$, by definition of $\rho^{k}$, and $\alpha \leq$ $\rho(\boldsymbol{x}, t) \leq \beta$ in $(\boldsymbol{x}, t) \in Q$, from (4). Then the above Sobolev embedding shows $\alpha-\frac{\alpha}{2} \leq \rho^{k}(\boldsymbol{x}, t) \leq \beta+\frac{\alpha}{2}:=\widetilde{\beta}$ in $\Omega \times\left[0, t^{*}\right]$, due to $\left\|\rho(\boldsymbol{x}, t)-\rho^{k}(\boldsymbol{x}, t)\right\|_{L^{\infty}(\Omega)} \leq \frac{\alpha}{2}$ in $\Omega \times\left[0, t^{*}\right]$. Therefore, (19) holds.

The following result states a priori estimates for $\left\|\nabla \boldsymbol{w}^{k}\right\|^{2}$ and $\left\|\Delta \psi^{k}\right\|$. 
Lemma 16. Suppose that there exist a constant $L>0$ and a noninteger $k \in$ $\mathbb{N}$ in such a way that $\mu\left\|\nabla \boldsymbol{w}^{k}\right\|^{2}+\left\|\Delta \psi^{k}\right\|^{2} \leq L\left(\frac{1}{\lambda_{k+1}}+\frac{1}{\tau_{k+1}}\right)$ and $\left\|\Delta e_{\rho}^{k}\right\|^{2} \leq$ $L\left(\frac{1}{\lambda_{k+1}}+\frac{1}{\tau_{k+1}}\right)$ hold on some interval $\left[0, t^{*}\right]$, for $L\left(\frac{1}{\lambda_{k+1}}+\frac{1}{\tau_{k+1}}\right)<\min \left\{\delta_{1}^{2}, \delta_{2}^{2},\right\}$ and $C_{\Omega}^{2} L\left(\frac{1}{\lambda_{k+1}}+\frac{1}{\tau_{k+1}}\right)<\frac{\alpha^{2}}{16}$. Then there exists a constant $K$ depending on the problem data such that

$$
\left\|A \boldsymbol{w}^{k}(t)\right\|^{2} \leq K, \quad\left\|\nabla \Delta \psi^{k}(t)\right\|^{2} \leq K
$$

for all $t \in\left[0, t^{*}\right]$.

Proof. From our assumptions in the lemma, it is easy to check the hypotheses in Lemma 15. Then, (19) holds. Now we draw on the definition of $\boldsymbol{w}^{k}=\boldsymbol{u}^{k}-\boldsymbol{v}^{k}$ and $\psi^{k}=\widetilde{\rho}^{k}-\delta^{k}$, and $\boldsymbol{v}^{k}$ and $\widetilde{\rho}^{k}$ are stable in $\boldsymbol{H}_{0}^{1}(\Omega)$ and $H_{N}^{3}(\Omega)$, by Lemma 2, respectively. Thus, one gets $\left\|\nabla \boldsymbol{u}^{k}(t)\right\| \leq\left\|\nabla \boldsymbol{v}^{k}(t)\right\|+\left\|\nabla \boldsymbol{w}^{k}(t)\right\| \leq M_{1}+\frac{1}{\mu} \min \left\{\delta_{1}, \delta_{2}\right\}$ and $\left\|\Delta \rho^{k}(t)\right\| \leq\left\|\Delta \delta^{k}(t)\right\|+\left\|\Delta \psi^{k}(t)\right\| \leq M_{2}+\min \left\{\delta_{1}, \delta_{2}\right\}$ for all $t \in\left[0, t^{*}\right]$. The next step is to embody $\left(\boldsymbol{u}^{k}, \rho^{k}\right)$ in the context of $(\boldsymbol{u}, \rho)$ in the proof of Lemma 7 to complete the result.

As mentioned above, our goal is to estimate $\boldsymbol{w}^{k}$ and $\psi^{k}$. To this end, we now want to make a comparison between $\boldsymbol{w}^{k}$ and $\boldsymbol{\xi}$, and, analogously, between $\psi^{k}$ and $\eta$. First, we state the equation for $\boldsymbol{w}^{k}$. Note that $\boldsymbol{v}^{k}$ satisfies

$$
\left\{\begin{array}{l}
\left(\rho \partial_{t} \boldsymbol{u}, \phi^{k}\right)+\left((\rho \boldsymbol{u} \cdot \nabla) \boldsymbol{u}, \phi^{k}\right)+\mu\left(\nabla \boldsymbol{v}^{k}, \nabla \boldsymbol{\phi}^{k}\right)-\lambda\left((\boldsymbol{u} \cdot \nabla) \nabla \rho, \boldsymbol{\phi}^{k}\right) \\
-\lambda\left((\nabla \rho \cdot \nabla) \boldsymbol{u}, \boldsymbol{\phi}^{k}\right)=\left(\rho \boldsymbol{f}, \boldsymbol{\phi}^{k}\right)
\end{array}\right.
$$

for all $\phi^{k} \in \boldsymbol{V}_{k}$, and $t \geq 0$. This is easily seen by using the weak formulation of (1) and considering the orthogonality relations for the $\left\{\boldsymbol{w}^{k}\right\}$. Subtracting (20) from (6) gives

$$
\left\{\begin{array}{l}
\left(\rho^{k} \partial_{t} \boldsymbol{w}^{k}, \boldsymbol{\phi}^{k}\right)+\mu\left(\nabla \boldsymbol{w}^{k}, \nabla \phi^{k}\right)=\left(\rho^{k} \partial_{t} \boldsymbol{e}_{\boldsymbol{u}}^{k}, \phi^{k}\right)+\left(\left(\rho-\rho^{k}\right)\left(\partial_{t} \boldsymbol{u}+\boldsymbol{u} \cdot \nabla \boldsymbol{u}-\boldsymbol{f}\right), \boldsymbol{\phi}^{k}\right) \\
+\left(\rho^{k} \boldsymbol{u} \cdot \nabla \boldsymbol{u}, \boldsymbol{\phi}^{k}\right)-\left(\rho^{k} \boldsymbol{u}^{k} \cdot \nabla \boldsymbol{u}^{k}, \boldsymbol{\phi}^{k}\right)-\lambda\left((\boldsymbol{u} \cdot \nabla) \nabla \rho, \boldsymbol{\phi}^{k}\right)+\lambda\left(\left(\boldsymbol{u}^{k} \cdot \nabla\right) \nabla \rho^{k}, \boldsymbol{\phi}^{k}\right) \\
-\lambda\left((\nabla \rho \cdot \nabla) \boldsymbol{u}, \boldsymbol{\phi}^{k}\right)+\lambda\left(\left(\nabla \rho^{k} \cdot \nabla\right) \boldsymbol{u}^{k}, \boldsymbol{\phi}^{k}\right),
\end{array}\right.
$$

which is the equation that $\boldsymbol{w}^{k}$ holds.

On the other hand, suppose that we fix a particular time $t_{0}$ as in Definition 3 , Then the weak variational form of (5) reads

$$
\left\{\begin{array}{l}
\left(\widehat{\rho} \partial_{t} \boldsymbol{\xi}, \boldsymbol{\phi}^{k}\right)+\left(\hat{\rho}(\boldsymbol{u} \cdot \nabla) \boldsymbol{\xi}, \boldsymbol{\phi}^{k}\right)+\left(\widehat{\rho}(\boldsymbol{\xi} \cdot \nabla) \boldsymbol{u}, \boldsymbol{\phi}^{k}\right)+\left(\widehat{\rho}(\boldsymbol{\xi} \cdot \nabla) \boldsymbol{\xi}, \boldsymbol{\phi}^{k}\right)+\mu\left(\nabla \boldsymbol{\xi}, \nabla \boldsymbol{\phi}^{k}\right) \\
-\lambda\left((\boldsymbol{\xi} \cdot \nabla) \nabla \rho, \boldsymbol{\phi}^{k}\right)-\lambda\left((\boldsymbol{\xi} \cdot \nabla) \nabla \eta, \boldsymbol{\phi}^{k}\right)-\lambda\left((\nabla \rho \cdot \nabla) \boldsymbol{\xi}, \boldsymbol{\phi}^{k}\right)-\lambda\left((\nabla \eta \cdot \nabla) \boldsymbol{\xi}, \boldsymbol{\phi}^{k}\right) \\
\left.-\lambda\left((\boldsymbol{u} \cdot \nabla) \nabla \eta, \boldsymbol{\phi}^{k}\right)-\lambda((\nabla \eta \cdot \nabla) \boldsymbol{u}), \boldsymbol{\phi}^{k}\right)=\left((\rho-\widehat{\rho})\left(\partial_{t} \boldsymbol{u}+\boldsymbol{u} \cdot \nabla \boldsymbol{u}-\boldsymbol{f}\right), \boldsymbol{\phi}^{k}\right)
\end{array}\right.
$$


for all $\boldsymbol{\phi}^{k} \in \boldsymbol{V}_{k}$, and $t \geq t_{0}$. Let us denote $\boldsymbol{\theta}=\boldsymbol{w}^{k}-\boldsymbol{\xi}$. Then subtracting (22) from (21) gives

$$
\left\{\begin{array}{l}
\left(\rho^{k} \partial_{t} \boldsymbol{\theta}, \boldsymbol{\phi}^{k}\right)+\mu\left(\nabla \boldsymbol{\theta}, \nabla \boldsymbol{\phi}^{k}\right)=\left(\rho^{k} \partial_{t} \boldsymbol{e}_{\boldsymbol{u}}^{k}, \boldsymbol{\phi}^{k}\right) \\
+\left(\left(\widehat{\rho}-\rho^{k}\right)\left(\partial_{t} \boldsymbol{u}+\boldsymbol{u} \cdot \nabla \boldsymbol{u}-\boldsymbol{f}+\partial_{t} \boldsymbol{\xi}\right), \boldsymbol{\phi}^{k}\right) \\
+\left(\rho^{k} \boldsymbol{u} \cdot \nabla \boldsymbol{u}, \boldsymbol{\phi}^{k}\right)-\left(\rho^{k} \boldsymbol{u}^{k} \cdot \nabla \boldsymbol{u}^{k}, \boldsymbol{\phi}^{k}\right) \\
+\lambda\left(\left(\boldsymbol{u}^{k} \cdot \nabla\right) \nabla \rho^{k}, \boldsymbol{\phi}^{k}\right)-\lambda\left((\boldsymbol{u} \cdot \nabla) \nabla \rho, \boldsymbol{\phi}^{k}\right) \\
+\lambda\left(\left(\nabla \rho^{k} \cdot \nabla\right) \boldsymbol{u}^{k}, \boldsymbol{\phi}^{k}\right)-\lambda\left((\nabla \rho \cdot \nabla) \boldsymbol{u}, \boldsymbol{\phi}^{k}\right) \\
+\left(\widehat{\rho}(\boldsymbol{u} \cdot \nabla) \boldsymbol{\xi}, \boldsymbol{\phi}^{k}\right)+\left(\widehat{\rho}(\boldsymbol{\xi} \cdot \nabla) \boldsymbol{u}, \boldsymbol{\phi}^{k}\right)+\left(\widehat{\rho}(\boldsymbol{\xi} \cdot \nabla) \boldsymbol{\xi}, \boldsymbol{\phi}^{k}\right) \\
-\lambda\left((\boldsymbol{\xi} \cdot \nabla) \nabla \rho, \boldsymbol{\phi}^{k}\right)-\lambda\left((\nabla \rho \cdot \nabla) \boldsymbol{\xi}, \boldsymbol{\phi}^{k}\right) \\
-\lambda\left((\boldsymbol{\xi} \cdot \nabla) \nabla \eta, \boldsymbol{\phi}^{k}\right)-\lambda\left((\nabla \eta \cdot \nabla) \boldsymbol{\xi}, \boldsymbol{\phi}^{k}\right) \\
\left.-\lambda\left((\boldsymbol{u} \cdot \nabla) \nabla \eta, \boldsymbol{\phi}^{k}\right)-\lambda((\nabla \eta \cdot \nabla) \boldsymbol{u}), \boldsymbol{\phi}^{k}\right) .
\end{array}\right.
$$

As our next step we estimate the right-hand side of (23), let us separate each pair of terms. To start with, we break up the convective terms as

$$
\begin{aligned}
\rho^{k} \boldsymbol{u} \cdot \nabla \boldsymbol{u}-\rho^{k} \boldsymbol{u}^{k} \cdot \nabla \boldsymbol{u}^{k}= & -\rho^{k} \boldsymbol{w}^{k} \cdot \nabla \boldsymbol{u}-\rho^{k} \boldsymbol{u} \cdot \nabla \boldsymbol{w}^{k} \\
& -\rho^{k} \boldsymbol{w}^{k} \cdot \nabla \boldsymbol{w}^{k}+\rho^{k} \boldsymbol{w}^{k} \cdot \nabla \boldsymbol{e}_{\boldsymbol{u}}^{k} \\
& +\rho^{k} \boldsymbol{e}_{\boldsymbol{u}}^{k} \cdot \nabla \boldsymbol{w}^{k}+\rho^{k} \boldsymbol{u} \cdot \nabla \boldsymbol{e}_{\boldsymbol{u}}^{k} \\
& +\rho^{k} \boldsymbol{e}_{\boldsymbol{u}}^{k} \cdot \nabla \boldsymbol{v}^{k}
\end{aligned}
$$

Next, we use $\boldsymbol{w}^{k}=P_{k} \boldsymbol{\theta}+P_{k} \boldsymbol{\xi}$ in the first three terms,

$$
\begin{gathered}
-\rho^{k}(\boldsymbol{u} \cdot \nabla) \boldsymbol{w}^{k}=-\rho^{k}(\boldsymbol{u} \cdot \nabla) P_{k} \boldsymbol{\theta}-\left(\rho^{k} \boldsymbol{u} \cdot \nabla\right) P_{k} \boldsymbol{\xi}, \\
-\rho^{k}\left(\boldsymbol{w}^{k} \cdot \nabla\right) \boldsymbol{u}=-\rho^{k}\left(P_{k} \boldsymbol{\theta} \cdot \nabla\right) \boldsymbol{u}-\rho^{k}\left(P_{k} \boldsymbol{\xi} \cdot \nabla\right) \boldsymbol{u}, \\
-\rho^{k}\left(\boldsymbol{w}^{k} \cdot \nabla\right) \boldsymbol{w}^{k}=-\rho^{k}\left(\boldsymbol{w}^{k} \cdot \nabla\right) P_{k} \boldsymbol{\theta}-\rho^{k}\left(\boldsymbol{w}^{k} \cdot \nabla\right) P_{k} \boldsymbol{\xi},
\end{gathered}
$$

and write the convective terms depending on the perturbation $\boldsymbol{\xi}$ by using the relation $\boldsymbol{\xi}=P_{k} \boldsymbol{\xi}+Q_{k} \boldsymbol{\xi}$ as

$$
\begin{aligned}
\widehat{\rho}(\boldsymbol{u} \cdot \nabla) \boldsymbol{\xi}= & \left(\widehat{\rho}-\rho^{k}\right)(\boldsymbol{u} \cdot \nabla) P_{k} \boldsymbol{\xi}+\rho^{k}(\boldsymbol{u} \cdot \nabla) P_{k} \boldsymbol{\xi}+\widehat{\rho}(\boldsymbol{u} \cdot \nabla) Q_{k} \boldsymbol{\xi}, \\
\widehat{\rho}(\boldsymbol{\xi} \cdot \nabla) \boldsymbol{u}= & \left(\widehat{\rho}-\rho^{k}\right)\left(P_{k} \boldsymbol{\xi} \cdot \nabla\right) \boldsymbol{u}+\rho^{k}\left(P_{k} \boldsymbol{\xi} \cdot \nabla\right) \boldsymbol{u}+\widehat{\rho}\left(Q_{k} \boldsymbol{\xi} \cdot \nabla\right) \boldsymbol{u}, \\
\widehat{\rho}(\boldsymbol{\xi} \cdot \nabla) \boldsymbol{\xi}= & \left(\widehat{\rho}-\rho^{k}\right)(\boldsymbol{\xi} \cdot \nabla) \boldsymbol{\xi}+\rho^{k}\left(\left(\boldsymbol{w}^{k}-P_{k} \boldsymbol{\theta}\right) \cdot \nabla\right) P_{k} \boldsymbol{\xi} \\
& +\rho^{k}\left(P_{k} \boldsymbol{\xi} \cdot \nabla\right) Q_{k} \boldsymbol{\xi}+\rho^{k}\left(Q_{k} \boldsymbol{\xi} \cdot \nabla\right) \boldsymbol{\xi},
\end{aligned}
$$

where in the second term on the right-hand side in the last equality we have used the fact that $P_{k} \boldsymbol{\theta}=\boldsymbol{w}^{k}-P_{k} \boldsymbol{\xi}$.

Now, combing the previous information about the convection terms, we obtain

$$
\begin{aligned}
g_{k}:= & \rho^{k}(\boldsymbol{u} \cdot \nabla) \boldsymbol{u}-\rho^{k}\left(\boldsymbol{u}^{k} \cdot \nabla\right) \boldsymbol{u}^{k}+\widehat{\rho}(\boldsymbol{u} \cdot \nabla) \boldsymbol{\xi}+\widehat{\rho}(\boldsymbol{\xi} \cdot \nabla) \boldsymbol{u}+\widehat{\rho}(\boldsymbol{\xi} \cdot \nabla) \boldsymbol{\xi} \\
= & \left(\widehat{\rho}-\rho^{k}\right)\left((\boldsymbol{u} \cdot \nabla) P_{k} \boldsymbol{\xi}+\left(P_{k} \boldsymbol{\xi} \cdot \nabla\right) \boldsymbol{u}+(\boldsymbol{\xi} \cdot \nabla) \boldsymbol{\xi}\right)+\rho^{k}\left(\boldsymbol{w}^{k} \cdot \nabla\right) \boldsymbol{e}_{\boldsymbol{u}}^{k} \\
& +\rho^{k}\left(\boldsymbol{e}_{\boldsymbol{u}}^{k} \cdot \nabla\right) \boldsymbol{w}^{k}+\rho^{k}(\boldsymbol{u} \cdot \nabla) \boldsymbol{e}_{\boldsymbol{u}}^{k}+\rho^{k}\left(\boldsymbol{e}_{\boldsymbol{u}}^{k} \cdot \nabla\right) \boldsymbol{v}^{k}-\rho^{k}(\boldsymbol{u} \cdot \nabla) P_{k} \boldsymbol{\theta} \\
& -\rho^{k}\left(P_{k} \boldsymbol{\theta} \cdot \nabla\right) \boldsymbol{u}-\rho^{k}\left(\boldsymbol{w}^{k} \cdot \nabla\right) P_{k} \boldsymbol{\theta}-\rho^{k}\left(P_{k} \boldsymbol{\theta} \cdot \nabla\right) P_{k} \boldsymbol{\xi}+\widehat{\rho}(\boldsymbol{u} \cdot \nabla) Q_{k} \boldsymbol{\xi} \\
& +\widehat{\rho}\left(Q_{k} \boldsymbol{\xi} \cdot \nabla\right) \boldsymbol{u}+\rho^{k}\left(P_{k} \boldsymbol{\xi} \cdot \nabla\right) Q_{k} \boldsymbol{\xi}+\rho^{k}\left(Q_{k} \boldsymbol{\xi} \cdot \nabla\right) \boldsymbol{\xi} .
\end{aligned}
$$


In order to treat the $\lambda$-terms, we observe that $\rho$ and $\rho^{k}$ can be changed to $\widetilde{\rho}$ and $\tilde{\rho}^{k}$ since the difference among them is up to an additive constant. Then

$$
\begin{aligned}
\lambda\left(\boldsymbol{u}^{k} \cdot \nabla\right) \nabla \rho^{k}-\lambda(\boldsymbol{u} \cdot \nabla) \nabla \rho= & \lambda\left(\boldsymbol{w}^{k} \cdot \nabla\right) \nabla \rho+\lambda(\boldsymbol{u} \cdot \nabla) \nabla \psi^{k} \\
& +\lambda\left(\boldsymbol{w}^{k} \cdot \nabla\right) \nabla \psi^{k}-\lambda\left(\boldsymbol{w}^{k} \cdot \nabla\right) \nabla e_{\rho}^{k} \\
& -\lambda\left(\boldsymbol{e}_{\boldsymbol{u}}^{k} \cdot \nabla\right) \nabla \psi^{k}-\lambda(\boldsymbol{u} \cdot \nabla) \nabla e_{\rho}^{k} \\
& -\lambda\left(\boldsymbol{e}_{\boldsymbol{u}}^{k} \cdot \nabla\right) \nabla \delta^{k} .
\end{aligned}
$$

Again, the relation $\boldsymbol{w}^{k}=P_{k} \boldsymbol{\theta}+P_{k} \boldsymbol{\xi}$ transforms the first term on the right-hand side:

$$
\lambda\left(\boldsymbol{w}^{k} \cdot \nabla\right) \nabla \rho=\lambda\left(P_{k} \boldsymbol{\theta} \cdot \nabla\right) \nabla \rho+\lambda\left(P_{k} \boldsymbol{\xi} \cdot \nabla\right) \nabla \rho .
$$

Now let us define $\pi=\psi^{k}-\eta$, then $\psi^{k}=G_{k} \pi+G_{k} \eta$. Therefore, the second and third term on the right-hand side is written as

$$
\begin{aligned}
\lambda(\boldsymbol{u} \cdot \nabla) \nabla \psi^{k} & =\lambda(\boldsymbol{u} \cdot \nabla) \nabla G_{k} \pi+\lambda(\boldsymbol{u} \cdot \nabla) \nabla G_{k} \eta, \\
\lambda\left(\boldsymbol{w}^{k} \cdot \nabla\right) \nabla \psi^{k} & =\lambda\left(\boldsymbol{w}^{k} \cdot \nabla\right) \nabla G_{k} \pi+\lambda\left(\boldsymbol{w}^{k} \cdot \nabla\right) G_{k} \eta .
\end{aligned}
$$

Finally, taking into account $\boldsymbol{\xi}=P_{k} \boldsymbol{\xi}+Q_{k} \boldsymbol{\xi}$ and $\eta=G_{k} \eta+H_{k} \eta$, we write the $\lambda$-terms depending on the perturbation $(\boldsymbol{\xi}, \eta)$ as

$$
\begin{gathered}
-\lambda(\boldsymbol{\xi} \cdot \nabla) \nabla \rho=-\lambda\left(P_{k} \boldsymbol{\xi} \cdot \nabla\right) \nabla \rho-\lambda\left(Q_{k} \boldsymbol{\xi} \cdot \nabla\right) \nabla \rho \\
-\lambda(\boldsymbol{u} \cdot \nabla) \nabla \eta=-\lambda(\boldsymbol{u} \cdot \nabla) \nabla G_{k} \eta-\lambda(\boldsymbol{u} \cdot \nabla) \nabla H_{k} \eta \\
-\lambda(\boldsymbol{\xi} \cdot \nabla) \nabla \eta=-\lambda\left(\left(\boldsymbol{w}^{k}-P_{k} \boldsymbol{\theta}\right) \cdot \nabla\right) \nabla G_{k} \eta-\lambda\left(P_{k} \boldsymbol{\xi} \cdot \nabla\right) \nabla H_{k} \eta-\lambda\left(Q_{k} \boldsymbol{\xi} \cdot \nabla\right) \nabla \eta
\end{gathered}
$$

Therefore, one gets

$$
\begin{aligned}
h_{k}:= & \lambda\left(\boldsymbol{u}^{k} \cdot \nabla\right) \nabla \rho^{k}-\lambda(\boldsymbol{u} \cdot \nabla) \nabla \rho-\lambda(\boldsymbol{\xi} \cdot \nabla) \nabla \rho-\lambda(\boldsymbol{\xi} \cdot \nabla) \nabla \eta-\lambda(\boldsymbol{u} \cdot \nabla) \nabla \eta \\
= & -\lambda\left(\boldsymbol{w}^{k} \cdot \nabla\right) \nabla e_{\rho}^{k}-\lambda\left(\boldsymbol{e}_{\boldsymbol{u}}^{k} \cdot \nabla\right) \nabla \psi^{k}-\lambda(\boldsymbol{u} \cdot \nabla) \nabla e_{\rho}^{k} \\
& -\lambda\left(\boldsymbol{e}_{\boldsymbol{u}}^{k} \cdot \nabla\right) \nabla \delta^{k}+\lambda\left(P_{k} \boldsymbol{\theta} \cdot \nabla\right) \nabla \rho+\lambda(\boldsymbol{u} \cdot \nabla) \nabla G_{k} \pi+\lambda\left(\boldsymbol{w}^{k} \cdot \nabla\right) \nabla G_{k} \pi \\
& -\lambda\left(Q_{k} \boldsymbol{\xi} \cdot \nabla\right) \nabla \rho-\lambda(\boldsymbol{u} \cdot \nabla) \nabla H_{k} \eta+\lambda\left(P_{k} \boldsymbol{\theta} \cdot \nabla\right) \nabla G_{k} \eta \\
& -\lambda\left(P_{k} \boldsymbol{\xi} \cdot \nabla\right) \nabla H_{k} \eta-\lambda\left(Q_{k} \boldsymbol{\xi} \cdot \nabla\right) \nabla \eta .
\end{aligned}
$$

The above treatment leads us to the following decomposition of the remaining $\lambda$-term:

$$
\begin{aligned}
i_{k}:= & \lambda\left(\nabla \rho^{k} \cdot \nabla\right) \boldsymbol{u}^{k}-\lambda(\nabla \rho \cdot \nabla) \boldsymbol{u}-\lambda(\nabla \rho \cdot \nabla) \boldsymbol{\xi}-\lambda(\nabla \eta \cdot \nabla) \boldsymbol{\xi}-\lambda(\nabla \eta \cdot \nabla) \boldsymbol{u} \\
= & -\lambda\left(\nabla e_{\rho}^{k} \cdot \nabla\right) \boldsymbol{w}^{k}-\lambda\left(\nabla \psi^{k} \cdot \nabla\right) \boldsymbol{e}_{\boldsymbol{u}}^{k}-\lambda\left(\nabla e_{\rho}^{k} \cdot \nabla\right) \boldsymbol{u} \\
& -\lambda\left(\nabla \delta^{k} \cdot \nabla\right) \boldsymbol{e}_{\boldsymbol{u}}^{k}+\lambda(\nabla \rho \cdot \nabla) P_{k} \boldsymbol{\theta}+\lambda\left(\nabla G_{k} \pi \cdot \nabla\right) \boldsymbol{u}+\lambda\left(\nabla G_{k} \pi \cdot \nabla\right) \boldsymbol{w}^{k} \\
& -\lambda(\nabla \rho \cdot \nabla) Q_{k} \boldsymbol{\xi}-\lambda\left(\nabla H_{k} \eta \cdot \nabla\right) \boldsymbol{u}+\lambda\left(\nabla G_{k} \eta \cdot \nabla\right) P_{k} \boldsymbol{\theta} \\
& -\lambda\left(\nabla H_{k} \eta \cdot \nabla\right) P_{k} \boldsymbol{\xi}-\lambda(\nabla \eta \cdot \nabla) Q_{k} \boldsymbol{\xi} .
\end{aligned}
$$

Next, the presence of the density-weighted time derivative for the velocity will reflect the difference between the Navier-Stokes and Kazhikhov-Smagulov equations. By applying the above process of factorization to (23) and taking $\boldsymbol{\phi}^{k}=P_{k} \partial_{t} \boldsymbol{\theta}$, we have

$$
\left(\rho^{k} \partial_{t} \boldsymbol{\theta}, P_{k} \partial_{t} \boldsymbol{\theta}\right)=\left\|\sqrt{\rho^{k}} \partial_{t} \boldsymbol{\theta}\right\|^{2}+\left(\rho^{k} \partial_{t} \boldsymbol{\theta}, Q_{k} \partial_{t} \boldsymbol{\xi}\right)
$$

since

$$
P_{k} \partial_{t} \boldsymbol{\theta}=\partial_{t} \boldsymbol{w}^{k}-\partial_{t} \boldsymbol{\xi}+Q_{k} \partial_{t} \boldsymbol{\xi}=\partial_{t} \boldsymbol{\theta}+Q_{k} \partial_{t} \boldsymbol{\xi}
$$


and

since

$$
\left(\nabla \boldsymbol{\theta}, \nabla P_{k} \partial_{t} \boldsymbol{\theta}\right)=\frac{1}{2} \frac{d}{d t}\left\|\nabla P_{k} \boldsymbol{\theta}\right\|^{2}
$$

$$
\begin{aligned}
\left(\nabla \boldsymbol{\theta}, \nabla P_{k} \partial_{t} \boldsymbol{\theta}\right) & =\left(\nabla\left(P_{k} \boldsymbol{\theta}+Q_{k} \boldsymbol{\theta}\right), \nabla P_{k} \partial_{t} \boldsymbol{\theta}\right) \\
& =\frac{1}{2} \frac{d}{d t}\left\|\nabla P_{k} \boldsymbol{\theta}\right\|^{2}-\left(\Delta Q_{k} \boldsymbol{\theta}, P_{k} \partial_{t} \boldsymbol{\theta}\right) \\
& =\frac{1}{2} \frac{d}{d t}\left\|\nabla P_{k} \boldsymbol{\theta}\right\|^{2}-\left(P \Delta Q_{k} \boldsymbol{\theta}, P_{k} \partial_{t} \boldsymbol{\theta}\right) \\
& =\frac{1}{2} \frac{d}{d t}\left\|\nabla P_{k} \boldsymbol{\theta}\right\|^{2}-\left(Q_{k} P \Delta \boldsymbol{\theta}, P_{k} \partial_{t} \boldsymbol{\theta}\right) \\
& =\frac{1}{2} \frac{d}{d t}\left\|\nabla P_{k} \boldsymbol{\theta}\right\|^{2} .
\end{aligned}
$$

Let us denote $j_{k}:=\partial_{t} \boldsymbol{u}+\boldsymbol{u} \cdot \nabla \boldsymbol{u}-\boldsymbol{f}+\partial_{t} \boldsymbol{\xi}+\boldsymbol{u} \cdot \nabla \boldsymbol{w}^{k}+\boldsymbol{w}^{k} \cdot \nabla \boldsymbol{u}+\boldsymbol{w}^{k} \cdot \nabla \boldsymbol{w}^{k}$. Finally, one obtains

$$
\begin{aligned}
\left\|\sqrt{\rho^{k}} \partial_{t} \boldsymbol{\theta}\right\|^{2}+\frac{\mu}{2} \frac{d}{d t}\left\|\nabla P_{k} \boldsymbol{\theta}\right\|^{2}= & \left(\rho^{k} \partial_{t} \boldsymbol{e}^{k}, P_{k} \partial_{t} \boldsymbol{\theta}\right)-\left(\rho^{k} \partial_{t} \boldsymbol{\theta}, Q_{k} \partial_{t} \boldsymbol{\xi}\right) \\
& +\left(\left(\widehat{\rho}-\rho^{k}\right) j_{k}+g_{k}+h_{k}+i_{k}, P_{k} \partial_{t} \boldsymbol{\theta}\right) .
\end{aligned}
$$

We now outline the estimate for the right-hand side of (24); for complete details, see [7]. To do so, we assume the regularity of $\boldsymbol{w}^{k}, \boldsymbol{\xi}, \psi^{k}$, and $\eta$ obtained in Lemmas 13 and 16. Also, Lemmas 1, 2, and 19 will be taken into account when needed. Indeed, given $\varepsilon>0$, we bound

$$
\begin{gathered}
\left(Q_{k} \partial_{t} \boldsymbol{\xi}, \rho^{k} \partial_{t} \boldsymbol{\theta}\right) \leq \varepsilon\left\|\partial_{t} \boldsymbol{\theta}\right\|^{2}+\frac{C_{\varepsilon}}{\lambda_{k+1}}\left\|\nabla \partial_{t} \boldsymbol{\xi}\right\|^{2}, \\
\left(\rho^{k} \partial_{t} \boldsymbol{e}^{k}, P_{k} \partial_{t} \boldsymbol{\theta}\right) \leq \varepsilon\left\|\partial_{t} \boldsymbol{\theta}\right\|^{2}+\leq \frac{C_{\varepsilon}}{\lambda_{k+1}}\left\|\nabla \partial_{t} \boldsymbol{u}\right\|^{2} .
\end{gathered}
$$

With an argument similar to that in [7, we estimate the convective terms as:

$$
\left(g_{k}, P_{k} \partial_{t} \boldsymbol{\theta}\right) \leq C_{\varepsilon}\left\|g_{k}\right\|^{2}+\varepsilon\left\|\partial_{t} \boldsymbol{\theta}\right\|^{2} \leq \frac{C_{\varepsilon}}{\lambda_{k+1}}+C_{\varepsilon}\left\|\nabla P_{k} \boldsymbol{\theta}\right\|^{2}+\varepsilon\left\|\partial_{t} \boldsymbol{\theta}\right\|^{2} .
$$

Just observe that the estimate for $\widehat{\rho}=\rho+\eta$ in the $L^{\infty}$-norm is needed to exploit the estimate in the $H^{2}$-norm of $\rho$ and $\eta$, from hypotheses (H2) and (H3), and the Sobolev embedding $H_{N, 0}^{2}(\Omega) \hookrightarrow L^{\infty}(\Omega)$. At this level we need not establish a maximum principle as in [7] for the density-dependent Navier-Stokes equations.

The idea to bound the $\lambda$-terms takes advantage of the extra regularity of the density. We may regard that $\nabla \rho$ plays the role of $\boldsymbol{u}$ and proceed as for the convective terms. Then we have

$$
\begin{aligned}
\left(h_{k}, P_{k} \partial_{t} \boldsymbol{\theta}\right) & \leq C_{\varepsilon}\left\|h_{k}\right\|^{2}+\varepsilon\left\|\partial_{t} \boldsymbol{\theta}\right\|^{2} \\
& \leq \frac{C_{\varepsilon}}{\lambda_{k+1}}+\frac{C_{\varepsilon}}{\tau_{k+1}}+C_{\varepsilon}\left\|\nabla P_{k} \boldsymbol{\theta}\right\|^{2}+\left\|\Delta G_{k} \pi\right\|^{2}+\varepsilon\left\|\partial_{t} \boldsymbol{\theta}\right\|^{2}, \\
\left(i_{k}, P_{k} \partial_{t} \boldsymbol{\theta}\right) & \leq C_{\varepsilon}\left\|i_{k}\right\|^{2}+\varepsilon\left\|\partial_{t} \boldsymbol{\theta}\right\|^{2} \\
& \leq \frac{C_{\varepsilon}}{\lambda_{k+1}}+\frac{C_{\varepsilon}}{\tau_{k+1}}+C_{\varepsilon}\left\|\nabla P_{k} \boldsymbol{\theta}\right\|^{2}+C_{\varepsilon}\left\|\Delta G_{k} \pi\right\|^{2}+\varepsilon\left\|\partial_{t} \boldsymbol{\theta}\right\|^{2} .
\end{aligned}
$$


Finally, we show how to estimate $j_{k}$. First, we decompose $\widehat{\rho}-\rho^{k}=\rho-\rho^{k}+\eta=$ $e_{\rho}^{k}-\psi^{k}+\eta=e_{\rho}^{k}-G_{k} \pi+H_{k} \eta$. Clearly, $\left\|j_{k}(t)\right\|$ is bounded, for all $t \in\left[0, t^{*}\right]$, by Lemmas 7, 13, and 16. Hence,

$$
\left(\left(\widehat{\rho}-\rho^{k}\right) j_{k}, P_{k} \partial_{t} \boldsymbol{\theta}\right) \leq \frac{C_{\varepsilon}}{\tau_{k+1}}+C_{\varepsilon}\left\|\Delta G_{k} \pi\right\|^{2}+\varepsilon\left\|\partial_{t} \boldsymbol{\theta}\right\|^{2} .
$$

The next step will be to repeat the same argument in the context of $\psi^{k}$ and of the perturbation for the density $\eta$. First, note that $\delta_{k}$ satisfies

$$
\left(\partial_{t} \delta^{k}, \nu^{k}\right)-\lambda\left(\Delta \delta^{k}, \nu^{k}\right)=-\left(\boldsymbol{u} \cdot \nabla \rho, \nu^{k}\right)
$$

for all $\nu^{k} \in D_{k}$. Next, subtracting the density equation from (7), this gives

$$
\left(\partial_{t} \psi^{k}, \nu^{k}\right)-\lambda\left(\Delta \psi^{k}, \nu^{k}\right)=\left(\boldsymbol{u} \cdot \nabla \rho, \nu^{k}\right)-\left(\boldsymbol{u}^{k} \cdot \nabla \rho^{k}, \nu^{k}\right) .
$$

Again, subtracting the perturbation equation for the density $(5)_{b}$ leads to

$$
\left\{\begin{array}{l}
\left(\partial_{t} \pi, \nu^{k}\right)-\lambda\left(\Delta \pi, \nu^{k}\right)=\left(\boldsymbol{u} \cdot \nabla \rho, \nu^{k}\right)-\left(\boldsymbol{u}^{k} \cdot \nabla \rho^{k}, \nu^{k}\right) \\
+\left(\boldsymbol{\xi} \cdot \nabla \rho, \nu^{k}\right)+\left(\boldsymbol{u} \cdot \nabla \eta, \nu^{k}\right)+\left(\boldsymbol{\xi} \cdot \nabla \eta, \nu^{k}\right),
\end{array}\right.
$$

where $\pi=\psi^{k}-\eta$ as before. Now, we are going to factorize the right-hand side of (26) as follows:

$$
\begin{aligned}
\boldsymbol{u} \cdot \nabla \rho-\boldsymbol{u}^{k} \cdot \nabla \rho^{k}= & -\boldsymbol{w}^{k} \cdot \nabla \rho-\boldsymbol{u} \cdot \nabla \psi^{k} \\
& -\boldsymbol{w}^{k} \cdot \nabla \psi^{k}+\boldsymbol{w}^{k} \cdot \nabla e_{\rho}^{k} \\
& +\boldsymbol{e}_{\boldsymbol{u}}^{k} \cdot \nabla \psi^{k}+\boldsymbol{u} \cdot \nabla e_{\rho}^{k} \\
& +\boldsymbol{e}_{\boldsymbol{u}}^{k} \cdot \nabla \delta^{k}
\end{aligned}
$$

As before, we break the first three terms on the right-hand side by using the fact that $\boldsymbol{w}^{k}=P_{k} \boldsymbol{\theta}+P_{k} \boldsymbol{\xi}$ and $\psi^{k}=G_{k} \pi+G_{k} \eta$ to get

$$
\begin{gathered}
-\boldsymbol{w}^{k} \cdot \nabla \rho=-P_{k} \boldsymbol{\theta} \cdot \nabla \rho-P_{k} \boldsymbol{\xi} \cdot \nabla \rho, \\
-\boldsymbol{u} \cdot \nabla \psi^{k}=-\boldsymbol{u} \cdot \nabla G_{k} \pi-\boldsymbol{u} \cdot \nabla G_{k} \eta, \\
-\boldsymbol{w}^{k} \cdot \nabla \psi^{k}=-\boldsymbol{w}^{k} \cdot \nabla G_{k} \pi-\boldsymbol{w}^{k} \cdot G_{k} \eta .
\end{gathered}
$$

By using the facts that $\boldsymbol{\xi}=P_{k} \boldsymbol{\xi}+Q_{k} \boldsymbol{\xi}$ and $\eta=G_{k} \eta+H_{k} \eta$, we get

$$
\begin{gathered}
\boldsymbol{\xi} \cdot \nabla \rho=P_{k} \boldsymbol{\xi} \cdot \nabla \rho+Q_{k} \boldsymbol{\xi} \cdot \nabla \rho, \\
\boldsymbol{u} \cdot \nabla \eta=\boldsymbol{u} \cdot \nabla G_{k} \eta+\boldsymbol{u} \cdot \nabla H_{k} \eta, \\
\boldsymbol{\xi} \cdot \nabla \eta=\left(\boldsymbol{w}^{k}-P_{k} \boldsymbol{\theta}\right) \cdot \nabla G_{k} \eta+P_{k} \boldsymbol{\xi} \cdot \nabla H_{k} \eta+Q_{k} \boldsymbol{\xi} \cdot \nabla \eta .
\end{gathered}
$$

Compiling the above information in (26), yields

$$
\begin{aligned}
\left(\partial_{t} \pi, \nu^{k}\right) & -\left(\lambda \Delta \pi, \nu^{k}\right) \\
= & -\left(\boldsymbol{w}^{k} \cdot \nabla e_{\rho}^{k}, \nu^{k}\right)-\left(\boldsymbol{e}_{\boldsymbol{u}}^{k} \cdot \nabla \psi^{k}, \nu^{k}\right)-\left(\boldsymbol{u} \cdot \nabla e_{\rho}^{k}, \nu^{k}\right) \\
& -\left(\boldsymbol{e}_{\boldsymbol{u}}^{k} \cdot \nabla \delta^{k}, \nu^{k}\right)+\left(P_{k} \boldsymbol{\theta} \cdot \nabla \rho, \nu^{k}\right)+\left(\boldsymbol{u} \cdot \nabla G_{k} \pi, \nu^{k}\right) \\
& +\left(\boldsymbol{w}^{k} \cdot \nabla G_{k} \pi, \nu^{k}\right)-\left(Q_{k} \boldsymbol{\xi} \cdot \nabla \rho, \nu^{k}\right)-\left(\boldsymbol{u} \cdot \nabla H_{k} \eta, \nu^{k}\right) \\
& +\left(P_{k} \boldsymbol{\theta} \cdot \nabla G_{k} \eta, \nu^{k}\right)+\left(P_{k} \boldsymbol{\xi} \cdot \nabla H_{k} \eta, \nu^{k}\right)-\left(Q_{k} \boldsymbol{\xi} \cdot \nabla \eta, \nu^{k}\right) .
\end{aligned}
$$


Now, take $\nu^{k}=-\Delta^{2} G_{k} \pi \in D_{k}$ in (27) and integrate by parts, and simple bounds yield

$\frac{1}{2} \frac{d}{d t}\left\|\Delta G_{k} \pi\right\|^{2}+\lambda\|\nabla \Delta \pi\|^{2} \leq \frac{C_{\varepsilon}}{\lambda_{k+1}}+\frac{C_{\varepsilon}}{\tau_{k+1}}+C_{\varepsilon}\left\|\nabla P_{k} \boldsymbol{\theta}\right\|^{2}+C_{\varepsilon}\left\|\Delta G_{k} \pi\right\|^{2}+\varepsilon\|\nabla \Delta \pi\|^{2}$.

In view of the above calculations and choosing $\varepsilon$ small enough we can state the following result.

Lemma 17. Suppose that there exist a constant $L>0$ and a noninteger $k \in$ $\mathbb{N}$ in such a way that $\mu\left\|\nabla \boldsymbol{w}^{k}\right\|^{2}+\left\|\Delta \psi^{k}\right\|^{2} \leq L\left(\frac{1}{\lambda_{k+1}}+\frac{1}{\tau_{k+1}}\right)$ and $\left\|\Delta e_{\rho}^{k}\right\|^{2} \leq$ $L\left(\frac{1}{\lambda_{k+1}}+\frac{1}{\tau_{k+1}}\right)$ hold on some interval $\left[0, t^{*}\right]$, for $L\left(\frac{1}{\lambda_{k+1}}+\frac{1}{\tau_{k+1}}\right)<\min \left\{\delta_{1}^{2}, \delta_{2}^{2}\right\}$ and $C_{\Omega}^{2} L\left(\frac{1}{\lambda_{k+1}}+\frac{1}{\tau_{k+1}}\right)<\frac{\alpha^{2}}{16}$. Let $(\boldsymbol{\xi}, \eta)$ be a perturbation of problem (11) defined in (5) and let $t_{0} \geq 0$ be a time such that $t_{0} \leq t^{*}$. Then we have

$$
\left\{\begin{array}{l}
\lambda\|\nabla \Delta \pi\|^{2}+\alpha\left\|\partial_{t} \boldsymbol{\theta}\right\|^{2}+\frac{d}{d t}\left\|\Delta G_{k} \pi\right\|^{2}+\mu \frac{d}{d t}\left\|\nabla P_{k} \boldsymbol{\theta}\right\|^{2} \\
\leq C\left\|\nabla P_{k} \boldsymbol{\theta}\right\|^{2}+C\left\|\Delta G_{k} \pi\right\|^{2}+\frac{C}{\lambda_{k+1}}\left(\left\|\nabla \partial_{t} \boldsymbol{\xi}\right\|^{2}+\left\|\nabla \partial_{t} \boldsymbol{u}\right\|^{2}\right)+\frac{C}{\lambda_{k+1}}+\frac{C}{\tau_{k+1}}
\end{array}\right.
$$

for all $t \in\left[t_{0}, t^{*}\right]$.

Next, by integrating (28) from $t$ to $t_{0}$, we arrive at

$$
\begin{aligned}
& \left\|\Delta G_{k} \pi(t)\right\|^{2}+\mu\left\|\nabla P_{k} \boldsymbol{\theta}(t)\right\|^{2}+\int_{t_{0}}^{t}\left(\widetilde{\alpha}\left\|\partial_{t} \boldsymbol{\theta}(\tau)\right\|^{2}+\lambda\|\nabla \Delta \pi(\tau)\|^{2}\right) \\
& \leq \mu\left\|P_{k} \boldsymbol{\theta}\left(t_{0}\right)\right\|^{2}+\left\|\Delta G_{k} \pi\left(t_{0}\right)\right\|^{2}+\frac{C}{\lambda_{k+1}} \int_{t_{0}}^{t}\left(\left\|\nabla \partial_{t} \boldsymbol{\xi}(\tau)\right\|^{2}+\left\|\nabla \partial_{t} \boldsymbol{u}(\tau)\right\|^{2}\right) d \tau \\
& +C \int_{t_{0}}^{t}\left(\left\|\nabla P_{k} \boldsymbol{\theta}(\tau)\right\|^{2}+\left\|\Delta G_{k} \pi(\tau)\right\|^{2}\right) d \tau+C\left(t-t_{0}\right)\left(\frac{1}{\lambda_{k+1}}+\frac{1}{\tau_{k+1}}\right) .
\end{aligned}
$$

Using Corollaries 8 and 14 in (29), one gets

$$
\begin{aligned}
& \left\|\Delta G_{k} \pi(t)\right\|^{2}+\mu\left\|\nabla P_{k} \boldsymbol{\theta}(t)\right\|^{2}+\int_{t_{0}}^{t}\left(\widetilde{\alpha}\left\|\partial_{t} \boldsymbol{\theta}(\tau)\right\|^{2}+\lambda\|\nabla \Delta \pi(\tau)\|^{2}\right) d \tau \\
& \leq \mu\left\|P_{k} \boldsymbol{\theta}\left(t_{0}\right)\right\|^{2}+\left\|\Delta G_{k} \pi\left(t_{0}\right)\right\|^{2}+C \int_{t_{0}}^{t}\left(\left\|\nabla P_{k} \boldsymbol{\theta}(\tau)\right\|^{2}+\left\|\Delta G_{k} \pi(\tau)\right\|^{2}\right) d \tau \\
& +C\left(\left(t-t_{0}\right)+1\right)\left(\frac{1}{\lambda_{k+1}}+\frac{1}{\tau_{k+1}}\right) .
\end{aligned}
$$

Finally, by applying Gronwall's Lemma, we estimate (30)

$$
\begin{aligned}
& \mu\left\|\nabla P_{k} \boldsymbol{\theta}(t)\right\|^{2}+\left\|\Delta G_{k} \pi(t)\right\|^{2}+\int_{t_{0}}^{t}\left(\widetilde{\alpha}\left\|\boldsymbol{\theta}_{t}\right\|^{2}+\lambda\|\nabla \Delta \pi(\tau)\|^{2}\right) d \tau \\
& \leq C_{1} e^{C_{1}\left(t-t_{0}\right)}\left(\mu\left\|P_{k} \boldsymbol{\theta}\left(t_{0}\right)\right\|^{2}+\left\|\Delta G_{k} \pi\left(t_{0}\right)\right\|^{2}+\left(\frac{1}{\lambda_{k+1}}+\frac{1}{\tau_{k+1}}\right)\left(\left(t-t_{0}\right)+1\right)\right) .
\end{aligned}
$$

From now on, we start with the proof of our main result. Let $T$ be sufficiently large such that $\frac{A_{1}^{2}}{\mu} F(T)^{2}+A_{2}^{2} G(T)^{2}<\frac{1}{8}$. Then, select $L_{1}:=16 C_{1} e^{C_{1} T}(T+1)$ and $L_{2}:=\max \left\{M_{3}, M_{4}\right\}$, where $M_{3}=\sup \|A \boldsymbol{u}(t)\|$ and $M_{4}=\sup _{t>0}\|\nabla \Delta \rho\|<\infty$, which are well defined by Lemma 7 . Let $L:=\max \left\{L_{1}, L_{2}\right\}$. Thus, one can find $N$ 
sufficiently large so that $L\left(\frac{1}{\lambda_{N+1}}+\frac{1}{\tau_{N+1}}\right)<\min \left\{\delta_{1}^{2}, \delta_{2}^{2}\right\}$ and $C_{\Omega}^{2} L\left(\frac{1}{\lambda_{N+1}}+\frac{1}{\tau_{N+1}}\right)$ $<\alpha^{2} / 16$. Next, we state that, for all $k \geq N$, we have

$$
\mu\left\|\nabla \boldsymbol{w}^{k}\right\|^{2}+\left\|\Delta \psi^{k}\right\|^{2}<L\left(\frac{1}{\lambda_{k+1}}+\frac{1}{\tau_{k+1}}\right) \text { for all } t \geq 0 .
$$

We proceed by contradiction. We suppose that (31) is false. Then there must exist $k \geq N$ and $t^{*}>0$ such that

$$
\mu\left\|\nabla \boldsymbol{w}^{k}\left(t^{*}\right)\right\|^{2}+\left\|\Delta \psi^{k}\left(t^{*}\right)\right\|^{2}=L\left(\frac{1}{\lambda_{k+1}}+\frac{1}{\tau_{k+1}}\right),
$$

where we have considered that $t^{*}$ appears for the first time in that condition. As an easy consequence of our election of $L$ and $N$ is that $\left\|\Delta\left(\rho(t)-\delta^{k}(t)\right)\right\|^{2} \leq$ $L\left(\frac{1}{\lambda_{k+1}}+\frac{1}{\tau_{k+1}}\right)$ holds for all $t \geq 0$, and $k \geq N$. Therefore, from (32), Lemmas [15] and 16 hold independently of $t^{*}$.

On one hand, we will first suppose that $t^{*} \leq T$. Then Lemma 17 also holds by taking $t_{0}=0, \boldsymbol{\xi}=0, \eta=0$. Thus, (30) becomes true. In particular, we see that $\left\|\nabla P_{k} \boldsymbol{\theta}\left(t_{0}\right)\right\|=0$ and $\left\|\Delta G_{k} \pi\left(t_{0}\right)\right\|=0$, and $\left\|\nabla P_{k} \boldsymbol{\theta}\right\|=\left\|\nabla \boldsymbol{w}^{k}\right\|$ and $\left\|\Delta G_{k} \pi\right\|=$ $\left\|\Delta \psi^{k}\right\|$. Therefore, we have

$$
\begin{aligned}
\mu\left\|\nabla \boldsymbol{w}^{k}\left(t^{*}\right)\right\|^{2}+\left\|\Delta \psi^{k}\left(t^{*}\right)\right\|^{2} & \leq C_{1} e^{C_{1} t^{*}}\left(t^{*}+1\right)\left(\frac{1}{\lambda_{k+1}}+\frac{1}{\tau_{k+1}}\right) \\
& \leq \frac{L_{1}}{16}\left(\frac{1}{\lambda_{k+1}}+\frac{1}{\tau_{k+1}}\right)
\end{aligned}
$$

whence

$$
\mu\left\|\nabla \boldsymbol{w}^{k}\left(t^{*}\right)\right\|^{2}+\left\|\Delta \psi^{k}\left(t^{*}\right)\right\|^{2}<L\left(\frac{1}{\lambda_{k+1}}+\frac{1}{\tau_{k+1}}\right),
$$

which contradicts (32). On the other hand, we will suppose that $t^{*}>T$. In this case, we take $t_{0}=t^{*}-T$, and $\boldsymbol{\xi}(t)$ and $\eta(t)$ satisfying $\boldsymbol{\xi}_{0}=\boldsymbol{w}^{k}\left(t_{0}\right)$ and $\eta_{0}=\psi^{k}\left(t_{0}\right)$. Again, inequality (30) is true by Lemma 17. Thus, one sees that

$$
\begin{aligned}
& \mu\left\|\nabla \boldsymbol{w}^{k}\left(t^{*}\right)-\nabla P_{k} \boldsymbol{\xi}\left(t^{*}\right)\right\|^{2}+\left\|\Delta \psi^{k}\left(t^{*}\right)-\Delta G_{k} \eta\left(t^{*}\right)\right\|^{2} \\
& \leq C_{1} e^{C_{1} T}(T+1)\left(\frac{1}{\lambda_{k+1}}+\frac{1}{\tau_{k+1}}\right)=\frac{L}{16}\left(\frac{1}{\lambda_{k+1}}+\frac{1}{\tau_{k+1}}\right) .
\end{aligned}
$$

Therefore, the triangular inequality gives

$$
\begin{aligned}
\mu\left\|\nabla \boldsymbol{w}^{k}\left(t^{*}\right)\right\|^{2}+\left\|\Delta \psi^{k}\left(t^{*}\right)\right\|^{2} \leq & 2\left(\left\|\nabla \boldsymbol{w}^{k}\left(t^{*}\right)-\nabla P_{k} \boldsymbol{\xi}\left(t^{*}\right)\right\|^{2}+\left\|\nabla P_{k} \boldsymbol{\xi}\left(t^{*}\right)\right\|^{2}\right) \\
& +2\left(\left\|\Delta \psi^{k}\left(t^{*}\right)-\Delta G_{k} \eta\left(t^{*}\right)\right\|^{2}+\left\|\Delta G_{k} \eta\left(t^{*}\right)\right\|^{2}\right) \\
\leq & 2\left(\frac{L}{16}\left(\frac{1}{\lambda_{k+1}}+\frac{1}{\tau_{k+1}}\right)+A_{1}^{2}\left\|\nabla \boldsymbol{\xi}\left(t_{0}\right)\right\|^{2} F(T)^{2}\right) \\
& +2\left(\frac{L}{16}\left(\frac{1}{\lambda_{k+1}}+\frac{1}{\tau_{k+1}}\right)+A_{2}^{2}\left\|\Delta \eta\left(t_{0}\right)\right\|^{2} G(T)^{2}\right) \\
\leq & 2\left(\frac{L}{4}\left(\frac{1}{\lambda_{k+1}}+\frac{1}{\lambda_{k+1}}\right)\right)=\frac{L}{2}\left(\frac{1}{\lambda_{k+1}}+\frac{1}{\tau_{k+1}}\right) \\
< & L\left(\frac{1}{\lambda_{k+1}}+\frac{1}{\tau_{k+1}}\right),
\end{aligned}
$$


where we have used the projection stability in Lemma 2 and the choice of $T$; but then we reach a contradiction (32).

Remark 18. Finally, we would like to extend this result to the complete model, i.e., considering the term $\lambda^{2} \nabla \cdot\left(\frac{1}{\rho} \nabla \rho \otimes \nabla \rho\right)$ on the left-hand side of $\mathbb{1}_{1}$. This introduces a slight modification in (5) 1 now being the equation

$$
\left\{\begin{array}{l}
\widehat{\rho} \partial_{t} \boldsymbol{\xi}+\widehat{\rho}(\boldsymbol{u} \cdot \nabla) \boldsymbol{\xi}+\widehat{\rho}(\boldsymbol{\xi} \cdot \nabla) \boldsymbol{u}+\widehat{\rho}(\boldsymbol{\xi} \cdot \nabla) \boldsymbol{\xi}-\mu \Delta \boldsymbol{\xi} \\
-\lambda((\boldsymbol{\xi} \cdot \nabla) \nabla \rho+(\boldsymbol{\xi} \cdot \nabla) \nabla \eta+(\boldsymbol{u} \cdot \nabla) \nabla \eta) \\
-\lambda((\nabla \rho \cdot \nabla) \boldsymbol{\xi}+(\nabla \eta \cdot \nabla) \boldsymbol{\xi}+(\nabla \eta \cdot \nabla) \boldsymbol{u})+\nabla q \\
+\lambda^{2} \nabla \cdot\left(\frac{1}{\widehat{\rho}}(\nabla \rho \otimes \nabla \eta+\nabla \eta \otimes \nabla \rho+\nabla \eta \otimes \nabla \eta)\right) \\
=-\eta\left(\partial_{t} \boldsymbol{u}+\boldsymbol{u} \cdot \nabla \boldsymbol{u}+\lambda^{2} \nabla \cdot\left(\frac{1}{\rho \widehat{\rho}}(\nabla \rho \otimes \nabla \rho)\right)-\boldsymbol{f}\right) .
\end{array}\right.
$$

Clearly, we may integrate by parts the $\lambda^{2}$-terms in the weak formulations (21) and (22), and treat them in a similar way to what was done for the convective term. Once we have established the analog to (23) we must again integrate by parts and use Leibniz' rule

$$
\lambda^{2} \nabla \cdot\left(\frac{1}{\kappa} \nabla \psi \otimes \nabla \zeta\right)=\lambda^{2} \frac{1}{\kappa}\left((\nabla \psi \cdot \nabla) \nabla \zeta-\frac{1}{\kappa} \nabla \kappa \cdot \nabla \psi \nabla \zeta+\nabla \zeta \Delta \psi\right) .
$$

This is due to the fact that we have no control of $\nabla P_{k} \partial_{t} \boldsymbol{\theta}$ on the left-hand side of (24). Next, all of the results derived in the paper remain valid, the only thing being that we need to use a lower bound for $\widehat{\rho}$ in order to control $\frac{1}{\hat{\rho}}$, and an estimate for $\nabla \widehat{\rho}$ and $\nabla \rho^{k}$ in the $L^{\infty}$-norm, which will appear in (24) as a consequence of the $\lambda^{2}$-terms. The control for $\nabla \widehat{\rho}$ in the $L^{\infty}$-norm comes by combining Lemmas 7 and 13, and the Sobolev embedding $H^{3}(\Omega) \hookrightarrow W^{1, \infty}(\Omega)$. For $\left(\boldsymbol{u}^{k}, \rho^{k}\right)$, we can state hypothesis $(H 2)$ on the time interval $\left[0, t^{*}\right]$ thanks to (32); therefore, Lemmal 7 holds, then the control for $\nabla \rho^{k}$ in the maximum norm. Finally, for the lower control of $\widehat{\rho}$ we recall that $\widehat{\rho}$ satisfies $\partial_{t} \widehat{\rho}-\lambda \Delta \widehat{\rho}+\boldsymbol{u} \cdot \nabla \widehat{\rho}=0$ in $\Omega \times\left(t_{0}, \infty\right)$ by Definition 3 , Thus, it suffices to show that $\widehat{\rho}\left(t_{0}\right)$ is bounded from below by a positive constant for our particular choice of perturbations. For the case $t^{*}<T$ we chose $t_{0}=0$ and $\eta=0$; therefore, $\widehat{\rho}=\rho$ which satisfies a maximum principle thanks to (4). Finally, for the case $t^{*}>T$, we selected $\eta_{0}=\psi^{k}\left(t_{0}\right)$ with $t_{0}=t^{*}-T$; therefore, $\hat{\rho}\left(t_{0}\right)-\rho\left(t_{0}\right)=\psi^{k}\left(t_{0}\right)$ by defintion. Next, from (32), we prove a pointwise estimate analogous to (19) by following its proof due to the fact that $\rho\left(t_{0}\right)$ satisfies (4).

Remark 19. The asymptotic behavior of the sequence of eigenvalues $\lambda_{k}$ and $\tau_{k}$ are described, for instance, in 22]. In that paper it is demonstrated that the behavior of eigenvalues for the Stokes and Laplacian operator is essentially the same. Then we can say that the rate of convergence obtained is optimal in the $\boldsymbol{H}^{1} \times H^{2}$-norm.

\section{Appendix A. Existence of approximate solutions}

Here we address the question of existence of approximate solutions for problem (6)-(7). We are inspired by the ideas developed in [13]. It is dealt with a linealization and fixed point argument. In order to keep the proof easy we divide it into four steps. The linealization of problem (6)-(7) reads as follows. 
(1) Given $\boldsymbol{w}_{*}^{k} \in C^{0}\left([0, \infty) ; \boldsymbol{V}_{k}\right)$, find $\rho_{*}^{k} \in C^{1}\left([0, \infty) ; D_{k}\right)$ such that

$$
\left(\partial_{t} \rho_{*}^{k}, \nu^{k}\right)+\left(\boldsymbol{w}_{*}^{k} \cdot \nabla \rho_{*}^{k}, \nu^{k}\right)-\lambda\left(\Delta \rho_{*}^{k}, \nu^{k}\right)=0 \quad \forall \nu^{k} \in D_{k}
$$

(2) Next, given $\boldsymbol{w}_{*}^{k} \in C^{0}\left([0, \infty) ; \boldsymbol{V}_{k}\right)$ and $\rho_{*}^{k} \in C^{1}\left([0, \infty) ; D_{k}\right)$, find $C^{1}\left([0, \infty) ; \boldsymbol{V}_{k}\right)$ such that

$$
\left\{\begin{array}{l}
\left(\rho_{*}^{k} \partial_{t} \boldsymbol{u}_{*}^{k}, \phi^{k}\right)+\left(\left(\rho_{*}^{k} \boldsymbol{w}_{*}^{k} \cdot \nabla\right) \boldsymbol{u}_{*}^{k}, \phi^{k}\right)+\mu\left(A \boldsymbol{u}_{*}^{k}, \phi^{k}\right)-\lambda\left(\left(\boldsymbol{u}_{*}^{k} \cdot \nabla\right) \nabla \rho_{*}^{k}, \boldsymbol{\phi}^{k}\right) \\
-\lambda\left(\left(\nabla \rho_{*}^{k} \cdot \nabla\right) \boldsymbol{u}_{*}^{k}, \boldsymbol{\phi}^{k}\right)=\left(\rho_{*}^{k} \boldsymbol{f}, \boldsymbol{\phi}^{k}\right) \quad \forall \boldsymbol{\phi}^{k} \in \boldsymbol{V}_{k} .
\end{array}\right.
$$

A.1. Existence for problem (34). To solve problem (34), we use the method of separation of variables. This technique reduces problem (34) to a system of ordinary differential equations. Indeed, write

$$
\rho_{*}^{k}(\boldsymbol{x}, t)=\sum_{j=1}^{k} \gamma_{j}(t) b_{j}(\boldsymbol{x})
$$

and note that problem (34) becomes the following linear differential system:

$$
\left\{\begin{array}{l}
\left.\sum_{\substack{j=1 \\
\gamma_{j}(0)}}^{k} a_{i j} \frac{d}{d t} \gamma^{j}(t)+\sum_{h=1}^{k} b_{i j}(t)\right]_{j},
\end{array}\right.
$$

where $a_{i j}=\delta_{i j}$, with $\delta_{i j}$ being the Kronecker delta, and

$$
b_{i j}(t)=\left(\boldsymbol{w}_{*}^{k}(t) \cdot \nabla b_{j}, b_{i}\right)+\lambda\left(\nabla b_{j}, \nabla b_{i}\right) \in C^{0}([0, \infty)),
$$

since $\boldsymbol{w}_{*}^{k} \in C^{0}\left([0, \infty) ; \boldsymbol{V}_{k}\right)$. Therefore, there exists a unique vector solution $\left\{\gamma^{j}(t)\right\}_{j=1}^{k}$ defined on $\left[0, t_{k}\right)$, in a neighborhood of $t=0$, satisfying (37). To prove that $t_{k}=\infty$, we test problem (34) by $\nu^{k}=\rho_{*}^{k}$ to get

$$
\frac{1}{2} \frac{d}{d t}\left\|\rho_{*}^{k}\right\|^{2}+\lambda\left\|\nabla \rho_{*}^{k}\right\|^{2}=0 .
$$

Next, if one integrates from 0 to $\infty$, one can assure that $t_{k}=\infty$ and obtain that $\rho_{*}^{k}$ is bounded in $C\left([0, \infty) ; L^{2}(\Omega)\right)$, independently of $\boldsymbol{w}_{*}^{k}$. Next, note that

$$
\sum_{j=1}^{k}\left|\gamma^{j}(t)\right|^{2}=\left\|\rho_{*}^{k}(t)\right\|^{2} \leq\left\|\rho_{0}\right\|^{2}
$$

since the eigenfunctions of the Neumann problem are orthonormal in the $L^{2}$-norm. Thus, there exists a unique solution $\rho_{*}^{k}$ bounded in $C\left([0, \infty) ; D_{k}\right)$, independently of $\boldsymbol{w}_{*}^{k}$, solving problem (34). The regularity properties of the coefficients of (37) imply that $\rho_{*}^{k}$ is bounded in $C^{1}\left([0, \infty) ; D_{k}\right)$ if $\boldsymbol{w}_{*}^{k}$ is bounded in $C^{0}\left([0, \infty) ; \boldsymbol{V}_{k}\right)$.

A.2. Approximate maximum principle for (34). Next, if we want to find a unique solution $\boldsymbol{u}_{*}^{k} \in C^{1}\left([0, \infty) ; \boldsymbol{V}_{k}\right)$ to problem (35), we need to prove a certain maximum principle for $\rho_{*}^{k}$. For this, we define the following auxiliary problem:

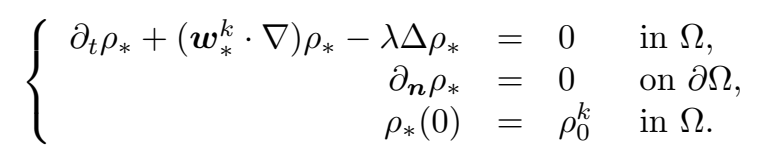


The key idea now is to obtain equation (27) but now in terms of $\rho_{*}$ and $\rho_{*}^{k}$. So, we need to introduce the concepts of perturbations to problem (38) similar to that of (5) 3 .

Definition 20. A function $\eta_{*}$ is called a perturbation of $\rho_{*}$, if $\widehat{\rho}_{*}=\rho_{*}+\eta_{*}$ is a solution of (38). Then, for a fixed $t_{0} \geq 0, \eta_{*}$ is a solution of the initial-boundary value problem

$$
\left\{\begin{array}{rlll}
\partial_{t} \eta_{*}+\left(\boldsymbol{w}_{*}^{k} \cdot \nabla\right) \eta_{*}-\lambda \Delta \eta_{*} & =0 & \text { in } \Omega \times\left(t_{0}, \infty\right), \\
\partial_{\boldsymbol{n}} \eta_{*} & =0 & \text { on } \partial \Omega \times\left(t_{0}, \infty\right), \\
\eta_{*}\left(t_{0}\right) & =\eta^{0} & \text { in } \Omega .
\end{array}\right.
$$

Since equation (38) is linear with respect to $\rho_{*}$, the expression of perturbation is much simpler than that of (5). The next lemma is proved by following Lemma 10.

Lemma 21. The perturbation $\eta_{*}$ is conditionally asymptotically stable, that is, there exist two positive numbers $\delta, A$ and a continuous decreasing function $F$ : $[0, \infty) \rightarrow \mathbb{R}^{+}$such that $F(0)=1$, and $\lim _{t \rightarrow \infty} F(t)=0$ so that for every $t_{0} \geq 0$ and for every $\eta_{*}^{0} \in H^{3}(\Omega)$ with $\partial_{n} \eta_{*}^{0}=0$ on $\Sigma$, satisfying $\left\|\Delta \eta_{*}^{0}\right\|<\delta_{2}$, problem (39) is uniquely solvable with

$$
\begin{gathered}
\eta \in L_{l o c}^{2}\left(\left[t_{0}, \infty\right) ; H_{N, 0}^{3}(\Omega)\right), \\
\partial_{t} \eta \in L_{l o c}^{2}\left(\left[t_{0}, \infty\right) ; H_{N, 0}^{2}(\Omega)\right) .
\end{gathered}
$$

Moreover,

$$
\left\|\Delta \eta_{*}(t)\right\| \leq A\left\|\Delta \eta_{*}^{0}\right\| F\left(t-t_{0}\right) \quad \forall t \geq t_{0}
$$

From now on, we choose the sequence $\left\{\boldsymbol{a}_{j}\right\}_{j=1}^{\infty}$ of eigenfunctions associated with the Stokes operator to be orthonormal with respect the Dirichlet norm. Let us write

$$
\boldsymbol{w}_{*}^{k}(\boldsymbol{x}, t)=\sum_{j=1}^{k} \varsigma_{j}(t) \boldsymbol{a}_{j}(\boldsymbol{x}) .
$$

Lemma 22. Let $\boldsymbol{w}_{*}^{k} \in C^{1}\left([0, \infty) ; \boldsymbol{V}_{k}\right)$. Assume that there exists a constant $W>0$ such that

$$
\sup _{t \geq 0} \sum_{j=1}^{k}\left|\varsigma^{j}(t)\right|^{2}=\sup _{t \geq 0}\left\|\nabla \boldsymbol{w}_{*}^{k}(t)\right\|^{2} \leq W
$$

Then it holds that

$$
\begin{gathered}
0<\alpha \leq \rho_{*}(\boldsymbol{x}, t) \leq \beta<\infty \text { in } Q \\
\sup _{t \geq 0}\left\|\Delta \rho_{*}(t)\right\| \leq W_{1}, \sup _{t \geq 0} e^{-\gamma t} \int_{0}^{t} e^{\gamma \tau}\left\|\nabla \Delta \rho_{*}(\tau)\right\|^{2} d \tau \leq W_{1},
\end{gathered}
$$

where $W_{1}>0$ is a constant depending on $\left\|\Delta \rho_{0}\right\|$ and $W$. Moreover, for all $t_{0}, t$ with $0 \leq t_{0} \leq t$, we have

$$
\int_{t_{0}}^{t}\left\|\nabla \Delta \rho_{*}(\tau)\right\|^{2} d \tau \leq C\left(t-t_{0}\right)+C
$$


Proof. Take $\nu^{k}=\rho_{*}^{k}$ in (35). Then one readily sees that

$$
\frac{1}{2} \frac{d}{d t}\left\|\rho_{*}^{k}\right\|^{2}+\lambda\left\|\nabla \rho_{*}^{k}\right\|^{2}=0
$$

In particular, we have $\sup _{t \geq 0}\left\|\rho_{*}^{k}(t)\right\| \leq\left\|\rho_{0}\right\|$. Now, let $\gamma>0$ be given. Multiply (44) by $e^{\gamma t}$ to get

$$
\frac{d}{d t}\left(e^{\gamma t}\left\|\rho_{*}^{k}\right\|^{2}\right)+2 \lambda e^{\gamma t}\left\|\nabla \rho_{*}^{k}\right\|^{2}=\gamma e^{\gamma t}\left\|\rho_{*}^{k}\right\|^{2} .
$$

Thus, integrating between 0 and $\tau$, one obtains

$$
e^{-\gamma t} \int_{0}^{t} e^{\gamma t}\left\|\nabla \rho_{*}^{k}(\tau)\right\|^{2} d \tau \leq C\left\|\rho_{0}\right\|^{2} .
$$

Next, pick $\nu^{k}=-\Delta \rho_{*}^{k}$ in (34). Hence we have

$$
\frac{d}{d t}\left\|\nabla \rho_{*}\right\|^{2}+\lambda\left\|\Delta \rho_{*}\right\|^{2} \leq C\left\|\nabla \boldsymbol{w}_{*}^{k}\right\|^{2} .
$$

The exponential technique together with (45) shows

$$
\left\|\nabla \rho_{*}\right\|^{2}+\lambda e^{-\gamma t} \int_{0}^{t} e^{\gamma \tau}\left\|\Delta \rho_{*}(\tau)\right\|^{2} d \tau \leq C\left(W+\left\|\nabla \rho_{0}\right\|^{2}\right) .
$$

On the other hand, test equation (34) by $\nu^{k}=-\Delta^{2} \rho_{*}^{k}$. Integration by parts and simple bounds show

$$
\frac{d}{d t}\left\|\Delta \rho_{*}\right\|^{2}+\lambda\left\|\nabla \Delta \rho_{*}\right\|^{2} \leq C\left\|\nabla \boldsymbol{w}_{*}^{k}\right\|^{4}\left\|\Delta \rho_{*}\right\|^{2} .
$$

From this, it is straightforward to complete the proof.

The proof of the following lemma is not hard by proceeding as in Lemma 22

Lemma 23. Under the conditions of Lemma 22 it holds that

$$
\sup _{t \geq 0}\left\|\Delta \eta_{*}(t)\right\| \leq C_{W}, \quad \sup _{t \geq 0} e^{-\gamma t} \int_{0}^{t} e^{\gamma \tau}\left\|\nabla \Delta \eta_{*}(\tau)\right\|^{2} d \tau \leq C_{W},
$$

where $C_{W}>0$ is a constant depending on $W$. Moreover, for all $t_{0}, t$ with $0 \leq t_{0} \leq t$, we have

$$
\int_{t_{0}}^{t}\left\|\nabla \Delta \eta_{*}(\tau)\right\|^{2} d \tau \leq C\left(t-t_{0}\right)+C
$$

Following the same method for obtaining equation (27), we get

$$
\left(\partial_{t} \pi_{*}, \nu^{k}\right)-\lambda\left(\Delta \pi_{*}, \nu^{k}\right)=\left(\boldsymbol{w}_{*}^{k} \cdot \nabla \rho_{*}, \nu^{k}\right)-\left(\boldsymbol{w}_{*}^{k} \cdot \nabla \rho_{*}^{k}, \nu^{k}\right)+\left(\boldsymbol{w}_{*}^{k} \cdot \nabla \eta_{*}, \nu^{k}\right),
$$

where we have defined $\pi_{*}=\psi_{*}^{k}-\eta_{*}$ with $\psi^{k}=\rho_{*}^{k}-\delta_{*}^{k}$, and $\delta_{*}^{k}=\sum_{n=1}^{k} d_{n}^{*}(t) b^{n}(\boldsymbol{x})$ being the orthogonal projection of $\rho_{*}$ onto $b_{1}, \ldots, b_{k}$, the first $k$ eigenfunction of the Poisson-Neumann operator $B$. We now decompose the right-hand side of (47) as follows:

$$
\begin{gathered}
\left(\boldsymbol{w}_{*}^{k} \cdot \nabla \rho_{*}, \nu^{k}\right)-\left(\boldsymbol{w}_{*}^{k} \cdot \nabla \rho^{k}, \nu^{k}\right)=-\left(\boldsymbol{w}_{*}^{k} \cdot \nabla \psi_{*}^{k}, \nu^{k}\right)+\left(\boldsymbol{w}_{*}^{k} \cdot \nabla e_{\rho_{*}}^{k}, \nu^{k}\right), \\
\left(\boldsymbol{w}_{*}^{k} \cdot \nabla \eta_{*}, \nu^{k}\right)=\left(\boldsymbol{w}_{*}^{k} \cdot \nabla G_{k} \eta_{*}, \nu^{k}\right)+\left(\boldsymbol{w}_{*}^{k} \cdot \nabla H_{k} \eta_{*}, \nu^{k}\right) .
\end{gathered}
$$


To complete the decomposition, the first term on the right-hand side of the first equality is treated as

$$
-\left(\boldsymbol{w}_{*}^{k} \cdot \nabla \psi_{*}^{k}, \nu^{k}\right)=-\left(\boldsymbol{w}_{*}^{k} \cdot \nabla G_{k} \pi_{*}, \nu^{k}\right)-\left(\boldsymbol{w}_{*}^{k} \cdot \nabla G_{k} \eta_{*}, \nu^{k}\right) .
$$

We thus get

$$
\left(\partial_{t} \pi_{*}, \nu^{k}\right)-\lambda\left(\Delta \pi_{*}, \nu^{k}\right)=\left(\boldsymbol{w}_{*}^{k} \cdot \nabla e_{\rho_{*}}^{k}, \nu^{k}\right)-\left(\boldsymbol{w}_{*}^{k} \cdot \nabla G_{k} \pi_{*}, \nu^{k}\right)+\left(\boldsymbol{w}_{*}^{k} \cdot \nabla H_{k} \eta_{*}, \nu^{k}\right) .
$$

Now, take $\nu^{k}=-\Delta^{2} G_{k} \pi \in D_{k}$ into (48) and integrate by parts. Direct calculations then lead to

$$
\frac{d}{d t}\left\|\Delta G_{k} \pi\right\|^{2}+\lambda\left\|\nabla \Delta G_{k} \pi\right\|^{2} \leq \frac{C_{\varepsilon}}{\tau_{k+1}^{1 / 2}}\left(\left\|\nabla \Delta \rho_{*}\right\|^{2}+\left\|\nabla \Delta \eta_{*}\right\|^{2}\right)+C_{\varepsilon}\left\|\Delta G_{k} \pi\right\|^{2} .
$$

Next, inequalities (43) and (46) applied to the above expression give

$$
\frac{d}{d t}\left\|\Delta G_{k} \pi\right\|^{2}+\lambda\left\|\nabla \Delta G_{k} \pi\right\|^{2} \leq C_{\varepsilon}\left\|\Delta G_{k} \pi\right\|^{2}+\frac{C_{\varepsilon}}{\tau_{k+1}^{1 / 2}}\left(\left(t-t_{0}\right)+1\right) .
$$

Finally, similar arguments to those for proving (31) show

$$
\left\|\Delta\left(\rho_{*}-\rho_{k}\right)\right\|^{2} \leq \frac{C}{\tau_{k+1}^{1 / 2}} .
$$

To conclude, we follow the ideas of Lemma 15 in proving (19) to deduce that there exists $k_{0}$ so that, for all $k \geq k_{0}$, we have

$$
0<\frac{\alpha}{2} \leq \rho_{*}^{k}(\boldsymbol{x}, t) \leq \widetilde{\beta} \text { in } Q .
$$

\section{A.3. Existence for problem (35). Write}

$$
\boldsymbol{u}_{*}^{k}(\boldsymbol{x}, t)=\sum_{j=1}^{k} \zeta_{j}(t) \boldsymbol{a}_{j}(\boldsymbol{x}) .
$$

Then problem (35) takes the form

$$
\left\{\begin{array}{l}
\sum_{j=1}^{k} a_{i j}(t) \frac{d}{d t} \zeta^{j}(t) \sum_{j=1}^{k} b_{i j}(t) \zeta^{j}(t)+c_{i}(t)=0 \text { in }(0, T), \text { for all } 1 \leq i \leq k, \\
\gamma_{j}(0)=\left[G_{h} \rho_{0}\right]_{j},
\end{array}\right.
$$

where

$$
\begin{gathered}
a_{i j}(t)=\left(\rho_{*}^{k}(t) \boldsymbol{a}_{i}, \boldsymbol{a}_{j}\right) \in C^{1}([0, \infty)), \\
b_{i j}(t)=\left(\left(\rho_{*}^{k}(t) \boldsymbol{w}_{*}^{k}(t) \cdot \nabla\right) \boldsymbol{a}_{j}(\boldsymbol{x}), \boldsymbol{a}_{i}\right)+\mu\left(\nabla \boldsymbol{a}_{j}, \nabla \boldsymbol{a}_{i}\right) \\
\left.-\lambda\left(\left(\boldsymbol{a}_{j} \cdot \nabla\right) \nabla\right) \rho_{*}^{k}(t), \boldsymbol{a}_{i}\right)-\lambda\left(\left(\nabla \rho_{*}^{k}(t) \cdot \nabla\right) \boldsymbol{a}_{j}, \boldsymbol{a}_{i}\right) \in C^{0}([0, \infty)),
\end{gathered}
$$

and

$$
c_{i}(t)=-\left(\rho_{*}^{k}(t) \boldsymbol{f}(t), \boldsymbol{a}_{i}\right) \in C^{1}([0, \infty))
$$

since $\boldsymbol{w}_{*}^{k} \in C^{0}\left([0, \infty) ; \boldsymbol{V}_{k}\right), \rho_{*}^{k} \in C^{1}\left([0, \infty) ; D_{k}\right)$, and $\boldsymbol{f} \in C^{1}\left([0, \infty) ; \boldsymbol{L}^{6 / 5}(\Omega)\right)$. The matrix $A=\left(a_{i j}\right)$ is uniformly positive defined with respect to time and simmetric. 
Indeed, from (49) we have

$$
\sum_{i, j=1}^{k} a_{i j}(t) \xi_{i} \xi_{j}=\int_{\Omega} \rho_{*}^{k}(t)\left(\sum_{i, j=1}^{k} \boldsymbol{a}_{i} \xi_{i}\right)^{2} \geq \widetilde{\alpha} \sum_{i=1}^{k}\left|\xi_{i}\right|^{2} .
$$

Therefore, the matrix $A$ is invertible, and hence, matricially, system (51) reads

$$
\left\{\begin{aligned}
\frac{d}{d t} \boldsymbol{\zeta}(t)+A^{-1}(t) B(t) \boldsymbol{\zeta}(t)+A^{-1}(t) \boldsymbol{c}(t) & =0 \text { in }(0, \infty), \\
\boldsymbol{\zeta}(0) & =H_{k} \boldsymbol{u}_{0} .
\end{aligned}\right.
$$

From the theory of ordinary differential equations we know that (52) has a unique vector solution $\boldsymbol{\zeta}(t)=\left\{\zeta^{j}(t)\right\}_{j=1}^{k}$ defined on $\left[0, t_{k}\right)$, in a neighborhood of $t=0$. In order to assure that $t_{k}=\infty$ we must derive some bounds for solutions of (35) independently of $k$. We are hampered by the nature of equations (34) and (35) to get a priori energy estimates for solutions of (35) because it requires equation (34) to be satisfied pointwisely. To understand this, one should notice that equation (34) may be written as

$$
\partial_{t} \rho_{*}^{k}+H_{k}\left(\boldsymbol{u}_{*}^{k} \cdot \nabla \rho_{*}^{k}\right)-\lambda \Delta \rho_{*}^{k}=0 \text { in } Q .
$$

Now, take $\phi^{k}=\boldsymbol{u}^{k}$ in (35) and multiply the above equation by $\frac{1}{2} \boldsymbol{u}_{*}^{k} \cdot \boldsymbol{u}_{*}^{k}$ and integrate over $\Omega$. Then we find, after adding the two equations, that there is no way of treating the sum

$$
\left(\left(\rho_{*}^{k} \boldsymbol{w}_{*}^{k} \cdot \nabla\right) \boldsymbol{u}_{*}^{k}, \boldsymbol{u}_{*}^{k}\right)+\frac{1}{2}\left(H_{k}\left(\boldsymbol{w}_{*}^{k} \cdot \nabla \rho_{*}^{k}\right), \boldsymbol{u}_{*}^{k} \cdot \boldsymbol{u}_{*}^{k}\right)
$$

which vanishes without the orthogonal projector $H_{k}$. Moreover, such energy estimates are obtained in [19] by imposing the constraint $\lambda<2 \mu /(\beta-\alpha)$ which has not appeared throughout this work. Hence we opt for estimates of the Dirichlet norm for the velocity rather than estimates of the energy norm. We proceed as follows. Let $\boldsymbol{\phi}^{k}=\partial_{t} \boldsymbol{u}_{*}^{k}$ in (35) and then $\phi^{k}=-A \boldsymbol{u}_{*}^{k}$ to obtain the following inequality, which is a variant of that in [5, 9, by using (42):

$$
\frac{d}{d t} \frac{\mu}{2}\left\|\nabla \boldsymbol{u}_{*}^{k}\right\|^{2}+\frac{\widetilde{\alpha}}{2}\left\|\partial_{t} \boldsymbol{u}_{*}^{k}\right\|^{2}+\frac{\widetilde{\alpha} \mu^{2}}{16 \widetilde{\beta}^{2}}\left\|A \boldsymbol{u}_{*}^{k}\right\|^{2} \leq C_{W_{1}}\left\|\nabla \boldsymbol{u}_{*}^{k}\right\|^{2}+C\|\boldsymbol{f}\|^{2} .
$$

Assuming that $C_{W_{1}}$ is smaller than for, e.g., $C_{P} \frac{\widetilde{\alpha} \mu^{2}}{32 \widetilde{\beta}^{2}} \leq C_{W_{1}}$ in equation (53) along with Poincaré's inequality $C_{P}\|\nabla \boldsymbol{v}\| \leq\|A \boldsymbol{v}\|$ for all $\boldsymbol{v} \in \boldsymbol{H}^{2}(\Omega) \cap \boldsymbol{V}$, it follows that

$$
\frac{d}{d t}\left\|\nabla \boldsymbol{u}_{*}^{k}\right\|^{2}+C_{P} \frac{\widetilde{\alpha} \mu}{32 \widetilde{\beta}^{2}}\left\|\nabla \boldsymbol{u}_{*}^{k}\right\|^{2} \leq C\|\boldsymbol{f}\|^{2} .
$$

Then one can easily see by multiplying (54) by $e^{\lambda t}$ with $\lambda=C_{P} \frac{\widetilde{\alpha} \mu}{32 \widetilde{\beta}^{2}}$ that $t_{k}=\infty$ and $\boldsymbol{u}_{*}^{k}$ is bounded in $C\left([0, \infty) ; \boldsymbol{H}_{0}^{1}(\Omega)\right)$, dependently on $\boldsymbol{w}_{*}^{k}$. Next, observe that

$$
\sum_{j=1}^{k}\left|\zeta^{j}(t)\right|^{2}=\left\|\nabla \boldsymbol{w}_{*}^{k}(t)\right\|^{2} \leq\left\|\nabla \boldsymbol{u}_{0}\right\|^{2}+C \sup _{t \leq 0}\|\boldsymbol{f}(t)\|^{2},
$$

since the eigenfunctions of the Stokes operator are orthonormal in the $\boldsymbol{H}_{0}^{1}$-norm. Thus, there exists a unique solution $\boldsymbol{u}_{*}^{k}$ bounded in $C^{0}\left([0, \infty) ; D_{k}\right)$ solving problem (35).

Again, the regularity properties of the coefficients of (51) imply that $\boldsymbol{u}_{*}^{k}$ is bounded in $C^{1}\left([0, \infty) ; \boldsymbol{V}_{k}\right)$ if $\boldsymbol{w}_{*}^{k}$ is bounded in $C^{0}\left([0, \infty) ; \boldsymbol{V}_{k}\right)$. 
Remark 24. The smallness of the constant $C_{W_{1}}$ in equation (53) implies $W_{1}$ to be small in Lemma 22. As a consequence of this, we have that $\left\|\Delta \rho_{0}\right\|$ and $W$ in Lemma 22 must be small.

A.4. Fixed point argument. So far, we know that if $\boldsymbol{w}_{*}^{k}$ is such that $\left\|\boldsymbol{w}_{*}^{k}\right\|_{C_{b}\left([0, \infty) ; \boldsymbol{V}_{k}\right)} \leq W$, there exists a positive constant $C_{W}$ such that $\boldsymbol{u}_{*}^{k}$ solution of (34) satisfies $\left\|\boldsymbol{u}_{*}^{k}\right\|_{C_{b}^{1}\left([0, \infty) ; \boldsymbol{V}_{k}\right)} \leq C_{W}$. We further show that for $\left\|\nabla \boldsymbol{u}_{0}\right\|^{2}+$ $C \sup _{t \leq 0}\|\boldsymbol{f}(t)\|^{2} \leq W$ the solution $\boldsymbol{u}_{*}^{k}$ of (34) fulfills $\left\|\boldsymbol{u}_{*}^{k}\right\|_{C_{b}\left([0, \infty) ; \boldsymbol{V}_{k}\right)} \leq W$ as observed in (55). If we define $B$ to be the closed ball with radius $W$ in $C_{b}^{1}\left([0, \infty) ; \boldsymbol{V}_{k}\right)$ and $B_{1}$ to be the closed ball with radius $C_{W}$ in $C_{b}^{1}\left([0, \infty) ; \boldsymbol{V}_{k}\right)$, then the mapping

$$
T: \boldsymbol{w}_{*}^{k} \in B \subset C_{b}\left([0, \infty) ; \boldsymbol{V}_{k}\right) \rightarrow T \boldsymbol{w}_{*}^{k}=\boldsymbol{u}_{*}^{k} \in B_{1} \subset C_{b}^{1}\left([0, \infty) ; \boldsymbol{V}_{k}\right)
$$

is continuous. It is clear from the continuous dependence on parameters of ordinary differential equations. A modified version of the Ascoli-Arzelà theorem guarantees compactness from $C_{b}^{1}\left([0, \infty) ; \boldsymbol{V}_{k}\right)$ to $C_{b}\left([0, \infty) ; \boldsymbol{V}_{k}\right)$. Having said this, the Shauder fixed-point theorem finally assures that there exists a fixed-point $\boldsymbol{u}^{k}$ of the above mapping belonging to $C_{b}^{0}\left([0, \infty) ; \boldsymbol{V}_{k}\right)$. Moreover, we can say that $\left(\boldsymbol{u}^{k}, \rho^{k}\right) \in C^{2}\left([0, \infty) ; \boldsymbol{V}_{k} \times D_{k}\right)$ by construction of solutions. Therefore, existence of solutions for problems (6) and (77) is completed.

Remark 25. Existence of solutions for problem (6)-(7) is presented under the hypothesis of smallness on the data problem $\boldsymbol{u}_{0}, \rho_{0}$, and $\boldsymbol{f}$. To be able to avoid such a restriction, we must choose the finite-dimensional spaces $\boldsymbol{V}_{k}$ and $D_{k}$ to satisfy the compatibility condition $\boldsymbol{V}_{k} \cdot \boldsymbol{V}_{k} \subset D_{k}$. The finite element method seems to be the appropriate context in which to define the spaces $\boldsymbol{V}_{k}$ and $D_{k}$. In other words, the approximating finite element spaces are made of piecewise polynomial functions. So, choosing $\boldsymbol{V}_{k}$ and $D_{k}$ as $\mathcal{P}_{k}$ and $\mathcal{P}_{2 k}$ Lagrange finite element space on a triangulation of $\Omega$, respectively, the condition $\boldsymbol{V}_{k} \cdot \boldsymbol{V}_{k} \subset D_{k}$ is easily satisfied.

\section{ACKNOWLEDGEMENTS}

We thank the anonymous reviewers for their insightful comments and suggestions which have contributed to improve the original version of this work.

\section{REFERENCES}

[1] C. Amrouche, V. Girault, On the existence and regularity of the solution of Stokes problem in arbitrary dimension. Proc. Japan Acad. Ser. A Math. Sci.67 (1991), no. 5, 171-175. MR.1114965 (92i:35098)

[2] S. N. Antoncev, A. V. Kazhikov, V. N. Monakhov, Boundary value problems in mechanics of nonhomogeneous fluids, North-Holland, 1990. MR1035212 (91d:76018)

[3] H. Beirão da Veiga, R. Serapioni, A, Valli, On the motion of nonhomogeneous fluids in the presence of diffusion. J. Math. Anal. Appl. 85 (1982), no. 1, 179-191. MR647566 (84a:76042)

[4] H. Beirão da Veiga,Diffusion on viscous fluids. Existence and asymptotic properties of solutions, Ann. Sc. Norm. Sup. Pisa, 10 (1983), 341-355. MR728440 (86c:76051)

[5] H. Beirão da Veiga, Long time behaviour of the solutions to the Navier-Stokes equations with diffusion, Nonlinear Anal., vol. 27, no. 11, 1996, 1229-1239. MR1408868(97h:35180)

[6] J.L. Boldrini, M.A. Rojas-Medar, An error estimate uniform in time for spectral semiGalerkin approximations of the nonhomogeneous Navier-Stokes equations, Numer. Funct. Anal. Optim. 15 (1994), no. 7-8, 755-778. MR.1305572 (95i:65131) 
[7] P. Braz e Silva, M.A. Rojas-Medar, Error bounds for semi-Galerkin approximations of nonhomogeneous incompressible fluids, Journal of Mathematical Fluid Mechanics, Volume 11, Number 2 / June, 2009, 186-207. MR2516131 (2010e:65154)

[8] P. D. Damázio, M. A. Rojas-Medar, On the convergence rate of semi-Galerkin approximations for the equations of viscous fluids in the presence of diffusion, Mat. Contemp. 15 1998, 105125. MR1661647 (99m:76092)

[9] P. D. Damázio, F. Guillén-González, J. V. Gutiérrez-Santacreu, M. A. Rojas-Medar, Local and global strong solution by the semi-Galerkin method for the model of mass diffusion. Mat. Contemp. 32 (2007), 63-83. MR2428427 (2009i:35242)

[10] D. A. Frank, V. I. Kamenetskii, Diffusion and Heat Transfer in Chemical Kinetics, Plenum Press, 1969.

[11] F. Guillén-González, Sobre un modelo asintótico de difusión de masa para fluidos incompresibles, viscoso y no homogéneos. Prooceedings of the Third Catalan Days On Applied Mathematics (1996) 103-114, ISBN: 84-87029-87-6.

[12] F. Guillén-González, P. Damásio, M. A. Rojas-Medar, Approximation by an iterative method for regular solutions for incompressible fluids with diffusion. Journal of Mathematical Analysis and Applications, 326, 1 (2007), 468-487. MR2277796 (2007m:76104)

[13] F. Guillén-González, J.V. Gutiérrez-Santacreu, Conditional stability and convergence of a fully discrete scheme for 3D Navier-Stokes equations with mass diffusion. SIAM J. Numer. Anal. 46, 5 (2008), 2276-2308. MR2421036 (2010d:65250)

[14] J.G. Heywood, The Navier-Stokes equations: on the existence, regularity and decay of solutions. Indiana Univ. Math. J., 29, 5 (1980), 639-681. MR.589434 (81k:35131)

[15] J.G. Heywood, An error estimate uniform in time for spectral Galerkin approximations of the Navier-Stokes problem. Pacific J. Math., 98 (1982), 333-345. MR650014 (83i:65070)

[16] J.G. Heywood, R. Rannacher, Finite element aproximation of the nonstationary NavierStokes problem. I. Regularity of solutions and second order error estimates for spacial discretization. SIAM J. Num. Anal., 19, 2 (1982), 275-311. MR650052 (83d:65260)

[17] J.G. Heywood, R. Rannacher, Finite element aproximation of the nonstationary NavierStokes problem. II. Stability of solutions and error estimates uniform in time. SIAM J. Num. Anal., 23, 4 (1986), 750-777. MR849281(88b:65132)

[18] J.G. Heywood, R. Rannacher, An analysis of stability concepts for the Navier-Stokes equations, Journal für die reine und angewandte Mathematik, 372 (1986), 1-33. MR863516 (87k:35200)

[19] A. V. Kazhikov, Sh. Smagulov, The correctness of boundary-value problems in a diffusion model of inhomogeneous fluid. Dokl. Akad. Nauk. SSSR, 234 (1977), 330-332. MR0446059 (56:4391)

[20] O. A. Ladyzhenskaya, The mathematical theory of viscous incompressible fluid, Gordon and Breach, New York, 1969. MR0254401 (40:7610)

[21] V. Mikhailov, Équations aux Dérivées Partielles. Mir, Moscow, 1980. MR.595502(82a:35003b)

[22] G. Métivier, Valeurs propers d'opérateurs définies par la restriction de systèmes variationnels à des sous-espaces, J. Math. Pures et Appl., 57 (1978), 133-156. MR.505900 (81c:35100)

[23] R. Rautmann, On the convergence rate of nonstationary Navier-Stokes approximations, Proc. IUTAM Symp. 1979, Approximations Methods for Navier-Stokes Problem, R. Rautmann (Ed.), Springer-Verlag, Lect. Notes in Math., 771 (1980), 235-248. MR566012 (81c:35106)

[24] B. Rummler, The eigenfunctions of the Stokes operator in special domains. I, ZAMM 77 (1997) 8, 619-627. MR.1470712 (98f:35120)

[25] B. Rummler, The eigenfunctions of the Stokes operator in special domains. II, ZAMM 77 (1997) 9, 669-675. MR1472965 (98h:35192)

[26] B. Rummler, The eigenfunctions of the Stokes operator in special domains. III, ZAMM 82 (2002) 6, 399-407. MR1906228(2003f:35232)

[27] R. Salvi, On the existence of weak solutions of boundary-value problems in a diffusion model of an inhomogeneous liquid in regions with moving boundaries, Portugaliae Math. 43 (1986), 213-233. MR886668 (88h:35096)

[28] R. Salvi, Error estimates for the spectral Galerkin approximations of the solutions of NavierStokes type equations, Glasgow Math. J., 31 (1989), pp. 199-211. MR997818 (90f:35168)

[29] P. Secchi, On the initial value problem for the equations of motion of viscous incompressible fluids in the presence of diffusion, Bollettino U.M.I., 6 1-B, 1982, 1117-1130. MR683497 (84b:35104) 
[30] P. Secchi, On the motion of viscous fluids in the presence of diffusion, SIAM J. Math. Anal. 19 (1988), 22-31. MR 924542(89j:35109)

[31] R. Temam, Navier-Stokes equations, theory and numerical analysis, North-Holland, Amsterdam, 1979. MR603444 (82b:35133)

Departmento Matemática Aplicada I, Universidad de Sevilla, E. T. S. I. Informática. Avda. Reina Mercedes, s/N. 41012 Sevilla, Spain

E-mail address: juanvi@us.es

Grupo de Matemática Aplicada, Dpto. de Ciencias Básicas, Facultad de Ciencias, Universidad del Bío-Bío, Campus Fernando may, Casilla 447, Chillán, Chile

E-mail address: marko@ueubiobio.cl. 